\title{
1 Autophagy promotes photomorphogenesis during seedling development in Arabidopsis \\ 2 in carbon limiting conditions
}

3 Akila Wijerathna-Yapa ${ }^{1 \#}$, Santiago Signorelli ${ }^{1,2 \#}$, Ricarda Fenske ${ }^{1}$, Diep R. Ganguly ${ }^{3}$, Elke

4 Stroeher $^{1}$, Lei Li ${ }^{1,4}$, Barry J. Pogson ${ }^{3}$, Owen Duncan ${ }^{1}$, A. Harvey Millar ${ }^{1} *$

5 1. ARC Centre of Excellence in Plant Energy Biology, School of Molecular Science, The

6 University of Western Australia, 6009 Crawley, WA, Australia.

7 2. Laboratorio de Bioquímica, Departamento de Biología Vegetal, Facultad de Agronomía,

8 Universidad de la República, Uruguay.

9 3. ARC Centre of Excellence in Plant Energy Biology, Research School of Biology, The

10 Australian National University, Canberra, ACT, Australia.

11 4. Department of Plant Biology and Ecology, College of Life Sciences, Nankai University, 12300071 Tianjin, PR China.

13 \#Contributed equally, *Corresponding author harvey.millar@uwa.edu.au +61 864887245

Short title: Autophagy during photomorphogenesis in Arabidopsis

17 The author responsible for distribution of materials integral to the findings presented in this

18 article in accordance with the policy described in the Instructions for Authors

19 (www.plantcell.org) is: A. Harvey Millar (harvey.millar@uwa.edu.au). 


\section{Abstract}

23 Autophagy is a conserved catabolic process that plays an essential role under nutrient 24 starvation condition and influences different developmental processes. We observed that seedlings of autophagy mutants (atg2, atg5, atg7, and atg9) germinated in the dark showed delayed chloroplast development following illumination. The delayed chloroplast development was characterized by a decrease in photosynthetic and chlorophyll biosynthetic proteins, lower chlorophyll content, reduced chloroplast size, and increased levels of proteins involved in lipid biosynthesis. Confirming the biological impact of these differences, photosynthetic performance was impaired in autophagy mutants $12 \mathrm{~h}$ post illumination. We investigated if the delayed chloroplast development could be explained by lower lipid import to the chloroplast or lower triglyceride (TAG) turnover. We observed that the limitations in the chloroplast lipid import imposed by trigalactosyldiacylglyceroll are unlikely to explain the delay in photomorphogenesis. However, we found that lower TAG mobility in the triacylglycerol lipase mutant sugardependent1 significantly affected photomorphogenesis. Moreover, we showed that lower levels of carbon resources exacerbated the delay in photomorphogenesis whereas higher levels of carbon resources had an opposite effect. This work provides evidence that autophagic process operate during de-etiolation in a manner that contributes to photomorphogenesis through increasing lipid turnover to physically or energetically sustain photomorphogenesis.

Keywords:Autophagy, atg, photomorphogenesis, chloroplast development, de-etiolation, lipids, lipophagy 


\section{Introduction}

Autophagy is a catabolic cellular process in which cytoplasmic content and organelles are degraded in lytic vacuoles (micro- and macro-autophagy) or in the cytosol (megaautophagy). In the latter, the degradation of cellular content is caused by the release of vacuolar lytic content in the cytosol leading to cell death. However, micro- and macroautophagy are processes contributing to cell maintenance and have been proved to be relevant in plant cell biology (Bassham 2007; Masclaux-Daubresse et al. 2017). Autophagy is performed by a series of autophagy-related (ATG) proteins which constitutes the autophagy core machinery, involved in the formation of autophagosomes and their fusion to lytic vacuoles (Marshall and Vierstra 2018).

Autophagic activity is known to be induced under certain conditions such as carbon and nitrogen starvation and to promote recycling of these elements (Avin-Wittenberg et al. 2015; Di Berardino et al. 2018; Luo et al. 2019). Due to this capacity, autophagy has been reported to be a key factor for developmental processes such as seed filling and maturation (Chung et al. 2009; Guiboileau et al. 2012; Janse van Rensburg et al. 2019). Autophagy can also be induced under a wide range of abiotic and biotic stress conditions, and its activity is known to contribute to plant stress tolerance (Avin-Wittenberg 2018; Signorelli et al. 2019).

In the past few years, sub-types of autophagy that influence specific cellular organelles have been reported, such as the mitophagy, chlorophagy, and pexophagy, among others, for the specific removal of mitochondria, chloroplasts and peroxisomes, respectively (Li et al. 2014; Young and Bartel 2016; Izumi et al. 2017). During seed germination and the early stages of seedling development, biogenesis of different organelles occurs and each play a role in metabolic transitions involved in utilising carbon resources from the seed and then from the atmosphere to sustain plant growth (Law et al. 2014; Pogson et al. 2015). During leaf senescence, selective degradation of organelles is reported to occur, linking mitophagy and chlorophagy (Izumi et al. 2010; Ishida et al. 2014; Broda et al. 2018).

In this work, first we used multiple reaction monitoring (MRM)-based targeted proteomics to assess the abundance of selected organelle proteins during early seedling development (i.e., seed reserve mobilisation), during dark-to-light transition of etiolated seedlings (i.e., photomorphogenesis) and light to dark transition of mature leaves (i.e., dark induced senescence) in different autophagy mutants and wild-type (WT). Our results highlight a chloroplast biogenesis delay in dark to light transition of etiolated seedling in autophagy mutants revealing new links between autophagy and i) the accumulation of 
photosynthetic machinery during photomorphogenesis, ii) the carbon status of seedlings and iii) plastid-dependent lipid mobilisation.

\section{$81 \quad$ Results}

Organelle abundance is selectively affected in autophagy mutants under different developmental transitions

We defined three different developmental transitions, early seedling development (T1), darkto-light greening of seedlings (T2), and light-to-dark senescence of leaves (T3), to investigate how organelle development processes are affected in autophagy mutants. To follow the differences in organelle development, we measured the abundance of organelle specific marker proteins in each developmental transition using a targeted proteomics (MRM) strategy, established in a previous study (Hooper et al. 2017). The protein markers used included key enzymes from the chloroplast, mitochondria, peroxisome, ER, Golgi, and plasma membrane, as well as subunits of the cytosolic ribosome. Among the studied markers, all the chloroplast proteins (PHOTOSYSTEM II SUBUNIT P-1, CHLOROPLAST RNA BINDING, LIGHT HARVESTING COMPLEX B6 OF PHOTOSYSTEM II and GERANYLGERANYL DIPHOSPHATE REDUCTASE) increased in abundance one day after the dark to light transition (Figure 1A-B), most were also induced in the early seedling development transition, but none of them changed in the light to dark senescence transition (Figure S1). When we analysed these organelle protein markers across the three developmental transitions in $\operatorname{atg} 2$, atg 5, atg7 and $\operatorname{atg} 9$, we observed all four atg lines showed a delay in the increase of chloroplast markers during dark to light transition. Chloroplast markers in most of the autophagy mutants were less abundant than WT at one day after light treatment (Figure 1C). Unlike the chloroplast markers, other organelles' protein markers showed no or inconsistent change during the dark to light transition (Figure S2). In the other two developmental transitions, no consistent differences were observed between atg lines and WT for any set of organelle markers (Supp. File 1 and 2).

Altered greening and chloroplast development occur in autophagy mutants transferred from dark to light conditions

We selected the dark to light transition to have a further understanding about the role of autophagy during the photomorphogenesis. Because the most significant differences between 
109 WT and autophagy mutants were observed at early stages of light exposure (Figure 1C), for

110 further experiments we focused on $0 \mathrm{~h}, 12 \mathrm{~h}$ and $24 \mathrm{~h}$ after exposure to light (Figure 2A). At

$1110 \mathrm{~h}$ all the seedlings were equally pale and presented a similar phenotype (Figure 2B-C);

112 however, at $12 \mathrm{~h}$, WT plants were significantly greener than all the autophagy mutants based

113 on visual inspection and calculation of a Green Index (GI) as outlined in the methods section

114 (Figure 2B-C). At 24h the GI of seedlings did not increase further in WT, suggesting they

115 already reached a maximum by $12 \mathrm{~h}$, whereas the autophagy mutants still increased their GI

116 over that time period (Figure 2B-C). Light treatment also increased the total chlorophyll

117 content measured in all the lines; however, WT plants had more chlorophyll than most

118 autophagy mutants, especially at 12h (Figure 2D). Furthermore, we analysed the chlorophyll

119 auto-fluorescence by confocal laser scanning microscopy as an indicator for presence and

120 size of chloroplasts (Figure 2E). We observed that at $12 \mathrm{~h}$ both the number of chloroplasts

121 per area and chloroplast size were reduced in autophagy mutants, whereas by $24 \mathrm{~h}$ most of the

122 differences between WT and autophagy mutants were lost (Figure 2F-G).

123

124

\section{Lower photosynthetic activity in de-etiolated seedlings of autophagy mutants}

To understand if the differences observed between autophagy mutants and WT in terms of chlorophyll content and chloroplast development have physiological consequences we evaluated the photosynthetic activity by different methods. First, we measured the chlorophyll fluorescence related parameters, maximum quantum efficiency of photosystem II $\left(\mathrm{F}_{\mathrm{V}} / \mathrm{F}_{\mathrm{M}}\right)$ and the quantum yield of non-regulated energy dissipation $(\boldsymbol{\varphi} \mathrm{NO})$. As observed in Figure $3 \mathrm{~A}$ there is a clear contrast between $\mathrm{F}_{\mathrm{V}} / \mathrm{F}_{\mathrm{M}}$ at $12 \mathrm{~h}$ and $24 \mathrm{~h}$ for all lines further confirming that at $12 \mathrm{~h}$ seedlings are not fully developed and have not reached their maximum photosynthetic capacity. At $12 \mathrm{~h}$, the $\mathrm{F}_{\mathrm{V}} / \mathrm{F}_{\mathrm{M}}$ was higher in WT than in the autophagy mutants (Figure 3B) indicating that WT plants were already more developed than autophagy mutants and thus made a more efficient use of incident light. However, at $24 \mathrm{~h} \mathrm{~F}_{\mathrm{V}} / \mathrm{F}_{\mathrm{M}}$ of autophagy mutants was more similar to WT values, suggesting that autophagy mutants eventually attained normal development. Likewise, $\boldsymbol{\varphi}$ NO values in autophagy mutants were more different to WT values at $12 \mathrm{~h}$ than at $24 \mathrm{~h}$, being always higher in autophagy mutants than WT values. We further investigated the relative apparent electron transport rate (ETR) parameter observing that in all lines the ETR values increased from $12 \mathrm{~h}$ to $24 \mathrm{~h}$ and in most cases the peak of ETR was shifted towards greater PAR from 12h to $24 \mathrm{~h}$ (Figure 3C). This finding suggests a greater photosynthetic performance and chloroplast development was 
evident by $24 \mathrm{~h}$ across all lines. However, WT ETRs were higher than ETR of autophagy mutants at both $12 \mathrm{~h}$ and $24 \mathrm{~h}$ and the WT peak was shifted to higher PAR values compared to autophagy mutants, which showed a more rapid decay at high light intensity (Figure 3C).

146 Finally, we tested the oxygen evolution of seedlings as an indicator of photosynthetic activity

147 and respiration, under light and dark conditions respectively. Respiration rates were similar 148 over the period of $24 \mathrm{~h}$ evaluated and among the different genotypes (Figure S4 A), however, 149 photosynthetic activity increased over the same period and varied between genotypes (Figure 150 S4 B) and so did the photosynthesis over respiration ratio (Figure 3D). The latter ratio is an indication of how photoautotrophic the seedlings were and thus of their developmental state. In particular, the photosynthesis over respiration ratio was similar between WT and autophagy mutants at $0 \mathrm{~h}$ and $24 \mathrm{~h}$ but significantly higher in WT than $\operatorname{atg} 5, \operatorname{atg} 7$ and $\operatorname{atg} 9$ at 12h (Figure 3D). Considering the different indicators for photosynthetic performance used here, we conclude that at $12 \mathrm{~h}$ the autophagy mutants had lower photosynthetic performance than WT, but the differences were diminished by $24 \mathrm{~h}$.

To better assess the differential chloroplast development in the autophagy mutants during the dark-light transition, we investigated the proteome of the autophagy mutants and WT plants at $0 \mathrm{~h}, 12 \mathrm{~h}$ and $24 \mathrm{~h}$ by quantitative mass spectrometry, and selected plastid-localized proteins for further analysis (Supp. File 3). We identified differentially enriched plastid proteins (DEPP) and subsequently the enrichment of specific metabolic pathways and biological process. Compared to WT, at $0 \mathrm{~h}$, the autophagy mutants $\operatorname{atg} 2$, atg 5 , atg 7 , and $\operatorname{atg} 9$ had lower abundance of 96, 83, 67, and 94 plastid proteins, respectively, and greater abundances of 32, 41, 47, and 25 plastid proteins, respectively. Among these DEPP, 51 lower abundance plastid proteins were common to all atg lines and 74 were common to at least three atg lines.

168 Likewise, 11 more abundant plastid proteins were common to all atg lines and 29 were common to at least three atg lines (Figure 4A). Similar patterns were observed at $12 \mathrm{~h}$ and

170 24h (Figure 4A). These data show a high consistency among the DEPP in the different autophagy mutants and across the timecourse (Figure 4B). In particular, we observed that 87

172 were more abundant with respect to WT in at least 3 atg lines in at least two time points. On

173 the other hand, 34 were more abundant than in WT in at least 3 atg lines and at two time 174 points. To explore these protein sets we analysed the GO biological process (GO BP) and 
175 KEGG pathway enrichment of this subset of consistent DEPP. The plastid proteins that were less abundant in at least 3 atg lines and at least two of the three times analysed showed an enrichment of proteins involved in photosynthesis and porphyrin and chlorophyll metabolism according to KEGG pathways, or an enrichment of chloroplast biogenesis, photosynthesis, and chlorophyll metabolism according to GO BP (Figure 4C). On the other hand, the more

180 abundant plastid proteins showed an enrichment of fatty acid biosynthesis according to KEGG pathway or an enrichment of fatty acid biosynthesis and sugar catabolism according to GO BP (Figure 4C).

184 We constructed a heatmap representing Z-scores of each family group of these DEPP set that were consistent over time and atg lines. The heatmap clearly showed two clusters, one of them including all the less abundant plastid proteins and the other one containing the more abundant plastid proteins (Figure 5). From the heatmap it is clear that the greatest differences in terms of less abundant plastid proteins are seen at $0 \mathrm{~h}$, and that atg9 is in general the least different to WT (Figure 5). The less abundant plastid proteins consisted of 86 proteins including 28 proteins related to photosynthesis; 12 proteins were related to the light phase of photosynthesis; 7 proteins were related to ATP synthesis; 6 were related to carbon fixation; 14 ribosomal proteins; 6 proteins involved in porphyrin and chlorophyll metabolism; and 6 proteins involved in amino acid metabolism (Figure 5).

The more abundant proteins were dominated by 8 proteins involved in carboxylic acid metabolism and biosynthesis, 8 proteins involved in carbohydrate metabolism and catabolism, 2 related to carbon fixation, and 2 involved in amine biosynthesis (Figure 5). Altogether, the lower abundance of proteins involved in photosynthesis and chlorophyll biosynthesis confirms the delayed chloroplast development in atg lines that has been suggested by the physiological data and the lower number and smaller chloroplast (Figure 2E) and the lower photosynthetic activity (Figure 3). The higher abundance sets of proteins indicate that the effect on altered chloroplast development in atg lines affect biochemical pathways differentially and do not simply imply less plastids or plastid size.

\section{The proteomic data is not explained by transcriptional regulation}

207 Given the high number of plastid proteins less abundant in autophagy mutants, we decided to investigate matched transcriptomics of these plants to see if there is an explanation for the 
DEPP set through altered transcriptional regulation. Therefore, we analysed transcriptomic data (Supp. File 4) for the subset of DEPP defined for Figure 5 and compared this data with

211 the equivalent proteomics data. However, there was no overall correlation between DEPPs

212 and their transcripts (Figure 6A). Nevertheless, it was clear that transcripts for less abundant

213 plastid proteins were typically more abundant at $12 \mathrm{~h}$ in atg lines than in WT, indicating that

214 lower protein abundances were not directly due to transcriptional downregulation in atg lines.

216 To further explore correlations, we used normalized proteomic and transcriptomic data and 217 performed Pearson correlation tests and evaluated their significance (Figure 6B). For the set 218 of less abundant plastid proteins in atg lines, there was a Pearson correlation between the 219 proteins and transcripts in all the cases (at a $p$-val=0.05, Figure 6B) but this correlation was often better for WT than for atg lines. For all the lines, the Pearson correlation was worse at Oh than at $12 \mathrm{~h}$ and $24 \mathrm{~h}$ (Figure 6B). Also, for this feature, atg9 was the one line that was most similar to WT (Figure 6B). The correlation for the more abundant proteins was lower than that for less abundant proteins (Figure 6B). In particular, atg2 and atg 5 never showed a significant correlation between protein and transcript abundances and only atg 9 showed a significant correlation at $0 \mathrm{~h}(p-\mathrm{val}=0.05)$ but not at $12 \mathrm{~h}$ and $24 \mathrm{~h}$ (Figure 6B). The WT showed a significant correlation at 12 and $24 \mathrm{~h}(p$-val=0.05) but not at $0 \mathrm{~h}$ (Figure 6B). At $p$ value of 0.01 , none of the lines showed a significant correlation.

Overall, the transcriptomic data highlights the importance of analysing proteomic data in autophagy mutants to gain understanding of the molecular pathways that are differentially expressed, as previously noted in maize autophagy mutant analysis (McLoughlin et al. 2018, 2020). In general, the results suggest that the transcriptional photomorphogenesis programs for plastid-localised gene products continues in autophagy mutants, and their proteomic differences are generally not explained by a differential transcription of the associated genes. To us, two main factors could be affecting the photomorphogenic process in atg lines, one could be the disruption of lipid membrane machinery that makes thylakoids ready to accept proteins, and the other could be an energetic restriction in atg lines that limits the translation/assembly of photosynthetic protein machinery. 
242 Autophagy mutants are known to have less triglyceride (TAG) and reduced TAG 243 mobilization (Fan et al 2019). Thus, we tested if a mutant for a triacylglycerol lipase, sugar 244 dependent 1 ( $s d p l$ ), not having less TAG but having impaired the capacity of using them (i.e, 245 reduced mobility), could mimic the greening phenotype of autophagy mutants. On the other 246 hand, if chloroplast biogenesis is slowed in autophagy mutants because they lack lipids to 247 build thylakoids, mutants for the chloroplast lipid permease trigalactosyldiacylglyceroll 248 (tgd1) might mimic the phenotype of atg lines, and tgdl/atg2 and tgdl/atg5 lines should further exacerbate the phenotype. Therefore, we also tested the phenotype of available tgdl single mutants and tgdlatg2 and tgdlatg5 double mutants (Fan et al 2019) during the dark to light transition to test these ideas.

252

253

254

255

256

257

258

259

260

261

262

263

264

265

266

267

268

269

270

271

272

273

The lipid metabolism mutant $s d p 1$ and double mutants $s d p 1 /$ atg 2 and $s d p 1 / a t g 5$ were affected in their ability to green after transfer from dark to light, even more than autophagy mutants (Figure 7A, Supp. Table 1). The lipid mutant tgdl and the double mutants tgdl/atg2 and tgd1/atg5 also showed lower GI compared to WT, but were not as affected as sdp1 mutants (Figure 7A, Supp. Table 1). In terms of the photosynthesis over respiration ratio, we observed that at $12 \mathrm{~h} s d p 1$ and double mutants $s d p 1 / a t g 2$ and sdp1/atg5 had lower values than WT plants and in some cases even lower values than autophagy mutants (Figure 7B). At 24h the differences persisted for the double mutants sdpl/atg2 and spdl/atg5 (Figure 7B). No differences were observed for tgdl single mutant at both 12 and 24h, and although the ratio was slightly lower for the double mutants tgd1/atg2 and tgdl/atg5, statistical differences were only found at $12 \mathrm{~h}$ for tgdl/atg5. Similar results were obtained in terms of maximum quantum yield of PSII. At 6 and $12 \mathrm{~h}$, all the single mutants atg2, atg5, sdpl and the double mutants spdl/atg2 spdlatg5 had lower $\mathrm{F}_{\mathrm{V}} / \mathrm{F}_{\mathrm{M}}$ values than $\mathrm{WT}$, and the differences persisted for the double mutants at $24 \mathrm{~h}$ (Figure 7C). However, the $\mathrm{F}_{\mathrm{V}} / \mathrm{F}_{\mathrm{M}}$ values for $\operatorname{tg} d 1$ were similar to WT at all times and only at $12 \mathrm{~h}$ were differences observed between the double mutants tgdl/atg2 and tgdl/atg5 (Figure 7C). Likewise, ETR was generally lower in autophagy mutants, sdpl and sdplatg2 and sdplatg5 double mutants (Figure 7D). However, tgdl, tgd1/atg2 and tgd1/atg5 mutants showed ETR values more similar to WT (Figure 7D).

Together, these results show that $\operatorname{tg} d l$ mutants mimick the greening phenotype of autophagy mutants but are not affected in terms of photosynthetic capacity, whereas the double mutants display very similar phenotypes to the atg single mutants (i.e. atg2 tgdl/atg2, and atg5 tgd1/atg5, Figure 7A-C, Supp. Table 1). Thus, for most of the parameters the addition 
of mutations in atg genes to $\operatorname{tgdl}$ mutants negatively affected its phenotype and almost mimicked its respective atg mutant (Figure 7B-C). This suggests that the atg mutations have more impact than the $\operatorname{tg} d 1$ mutation in photomorphogenesis. In contrast, $s d p 1$ mutants also displayed a delayed greening phenotype but it was clearly more pronounced than in the autophagy mutants (Figure 7, Table S1). The addition of mutations in atg genes to $s d p 1$ mutant did not rescue the phenotype, instead the phenotype was mostly similar to $s d p 1$ single mutants and worse than atg single mutants (Figure 7A, Supp. Table 1). This suggests that impaired mobility of TAG has a stronger effect on photomorphogenesis than the lack of autophagic activity, and also suggests that the reduced TAG mobility of atg mutants may contribute to their delay in photomorphogenesis.

\section{Lowered carbon availability exacerbates the delayed greening effect in autophagy mutants}

Given that our study on $s d p 1$ mutants suggested that the delay in photomorphogenesis of atg lines may be related to their reduced capacity to degrade TAG, we tested the greening phenotype under different levels of sucrose to see if lower levels of sucrose further compromised their phenotype and/or if higher levels of sucrose can attenuate their phenotype. We analysed the greening of WT and autophagy mutants during the dark to light transition $(0,6,12$ and $24 \mathrm{~h}$ post illumination) under normal conditions $(0.5 \% \mathrm{Suc})$, poor carbon source (C) conditions $(0.1 \%$ Suc) and rich C conditions (2\% Suc). At $0.5 \%$ Suc, the greening of the atg lines is delayed but all of them reach WT values by $24 \mathrm{~h}$ (Figure 8A). When subjected to the poor $\mathrm{C}$ medium the differences between WT and autophagy mutants were exacerbated, all of them being statistically different at 6 and $12 \mathrm{~h}$ and even one of them at $24 \mathrm{~h}$ (atg9), whereas when subjected to a richer C medium the differences with WT were completely lost (Figure 8A). Likewise, the lower $\mathrm{F}_{\mathrm{V}} / \mathrm{F}_{\mathrm{M}}$ of autophagy mutants observed at $6 \mathrm{~h}$ and $12 \mathrm{~h}$ in $0.5 \%$ sucrose are also observed in $0.1 \%$ sucrose but not in $2 \%$ sucrose (Figure 8B). As observed before, the differences in $\mathrm{F}_{\mathrm{V}} / \mathrm{F}_{\mathrm{M}}$ were reduced over the time becoming insignificant by $24 \mathrm{~h}$ except for $0.1 \%$ sucrose where some differences between WT and autophagy mutants persist (Figure 8B). The ETR was also reduced in autophagic mutants compared to WT plants, with the differences more significant at earlier times post illumination and at lower sucrose level (Figure 8C).

Altogether, the data show that a high level of sucrose can ameliorate the delay in greening observed in autophagy mutants confirming that $\mathrm{C}$ restrictions in autophagy mutants are one of the reasons why autophagy mutants display a delayed photomorphogenesis. 


\section{Discussion}

308 Autophagy mutants have delayed chloroplast development during photomorphogenesis

309 While the maturation from etioplast into chloroplast in cotyledons of seedlings during the

310 first hours after light exposure is a well-documented process (Rudowska et al. 2012; Pogson

311 et al. 2015), a role of autophagy in this process remains largely unexplored. When we found

312 that plastid organelle markers abundance was the most affected during the dark to light

313 transition of young autophagy mutant seedlings (Figure 1, Figure S1 and Figure S2), we

314 sought to further explore the role of autophagy in photomorphogenesis with a special focus

315 on chloroplast development.

316 Chloroplasts house the machinery of photosynthesis as well as nitrogen assimilation, fatty 317 acid and amino acid biosynthesis, among other process, thus their development is known to 318 strongly influence plant development (Pogson et al. 2015). Chloroplast development requires 319 multiple molecular processes such as nuclear gene expression, protein import, plastid gene 320 expression, chloroplast RNA processing and ribosome assembly, protein maturation and 321 degradation, thylakoid biogenesis, lipid biosynthesis, chlorophyll biosynthesis, metabolite 322 transport, and photosystem assembly (Waters and Langdale 2009). Here, we observed that autophagy mutants had lower chlorophyll content, a reduced number and size of chloroplasts

324 (Figure 2) and poorer light-phase dependent photosynthetic activity during photomorphogenesis (Figure 3), suggesting that one or more of these molecular processes involved in chloroplast development was affected in autophagy mutants. The lower PSII maximal efficiency in autophagy mutants, suggest either that the PSII is damaged or not fully developed at $12 \mathrm{~h}$ in these lines (Figure 3B), potentially due to lower chlorophyll levels in autophagy mutants at $12 \mathrm{~h}$ (Figure 2D). Moreover, at 12h, the higher $\boldsymbol{\varphi} \mathrm{NO}$ results observed

330 in autophagy mutants revealed that the photochemical energy conversion and protective regulatory mechanism in these mutants were less efficient than in the WT either due to a less active PSII reaction centre or a poorer trans-thylakoidal proton gradient (Figure 3B). As a consequence, the electron transport rate and photosynthetic activity in these mutants were lower than in WT (Figure 3C and D). Our data suggest that while autophagy mutants can complete photomorphogenesis, it is delayed compared to WT. From the literature it is known 
that by 3 to 5 weeks old, chlorophyll content is no longer reduced and there are no changes in the transcripts for chlorophyll biosynthesis and degradation in atg2 (Jiang et al. 2020).

Autophagy mutants show lower abundance of chlorophyll biosynthetic and photosynthetic proteins and increased abundance of proteins involved in plastid fatty acid biosynthesis

340 As autophagy acts post-transcriptionally to alter the protein complement of cells, it was important to study the abundance of plastid proteins to further understand the delayed greening identified in autophagy mutants. We observed that these mutants have lower abundance of proteins involved in chlorophyll biosynthesis and photosynthesis but greater abundance of proteins involved in fatty acid biosynthesis and carbohydrate metabolism (Figure 4 and Figure 5). These findings are in agreement with previous reports using adult maize autophagy mutants that showed reduced levels of thylakoidal proteins and increased levels of proteins involved in fatty acid metabolism (McLoughlin et al. 2018). Basal autophagy has been previously shown to contribute to TAG synthesis (Fan et al. 2019), thus it is possible that the observed increase in fatty acid biosynthetic proteins is a response to compensate for the lack of autophagy-mediated TAG synthesis. On the other hand, we observed that the differential abundance of proteins in autophagy mutants is not simply explained by transcriptional regulation (Figure 6). In general, the correlation at $0 \mathrm{~h}$ between protein and transcript abundance were poor for all genotypes (Figure 6B). At $12 \mathrm{~h}$ and $24 \mathrm{~h}$ after the transfer to light, the correlation for the less abundant proteins improved compared to Oh. However for the more abundant proteins, only WT showed a considerable improvement of the correlation between protein and transcript abundance at $12 \mathrm{~h}$ and $24 \mathrm{~h}$ compared to $0 \mathrm{~h}$, suggesting that some of these proteins may be degraded by autophagy in the WT plants but not in atg lines influencing the poorer correlation. Hence, it is unlikely that the differential expression of photosynthetic related proteins is related to disrupted signalling events in autophagy mutants resulting in lower transcription. Instead, it is more likely that the altered lipid metabolism in autophagy mutants affects the thylakoid structure and limits the assembly of photosynthetic complexes. A link between autophagy, photosynthesis and fatty acid has been established in the model alga Chlamydomonas reinhardtii, in which the inhibition of fatty acid biosynthesis was shown to downregulate photosynthesis and induce autophagy (Heredia-Martínez et al. 2018). In particular, the authors concluded that a change in the monogalactosyldiacyl-glycerols / digalactosyldiacyl-glycerols ratio (MGDG/DGDG) was responsible for altering the thylakoid structure and thus the photosynthetic activity (Heredia- 
Martínez et al. 2018). As autophagy is known to affect fatty acid content and composition in Arabidopsis (Minina et al. 2018; Fan et al. 2019; Havé et al. 2019), it is possible that the differing lipid profiles in autophagy mutants are partially responsible for the delayed chloroplast development and the resulting lower photosynthetic activity as observed in $C$. reinhardtii.

The delayed photomorphogenesis in autophagy mutants is probably not related to limitations in lipid import into the chloroplast or lower MGDG and DGDG content

375

TGD1 is a chloroplastic permease (inner envelope) involved in the unidirectional lipid transfer from the ER to plastids, necessary for thylakoids formation (Xu et al. 2003, 2010). The TGD complex is known to transport phosphatidylcholine (PC) but it is expected that other MGDG precursors are also transported (Michaud and Jouhet 2019). Potential candidates are phosphatidates (PA) and diacylglycerols (DAG) (LaBrant et al. 2018). These precursors are used to produce MGDG and DGDG which together compose $80 \%$ of chloroplast lipid and are essential for the light phase of photosynthesis ( $\mathrm{Li}$ and $\mathrm{Yu} 2018$ ) (Figure 9).

Because extensive lipid trafficking is required for thylakoid lipid biosynthesis (Awai et al. 2006) and the ER lipid assembly pathway was shown to be more relevant than the chloroplast pathway in the fatty acid biosynthesis and membrane lipid turnover under basal autophagy (Fan et al. 2019), we tested if $\operatorname{tgdl}$ mutants mimic the phenotype of autophagy mutants. It is worth noting that mature tgdl mutants do not show alterations in their photosynthetic parameters when compared to WT plants ( $\mathrm{Li}$ et al. 2012). While gross carbon assimilation were shown to be marginally affected (10-15\% less), Rubisco activity, electron transport rate and photosynthesis I and II oxidation status were not altered (Li et al. 2012). Hence, reduced photosynthetic capacity in tgdl during de-etiolation would suggest a developmental delay. Our results showed that $\operatorname{tg} d l$ mutants mimicked the slow greening phenotype of autophagy mutants (Figure 7A). Moreover, the $\operatorname{tg} d 1$ mutant is known to display a upregulation of the chloroplastic lipid biosynthetic pathway (Xu et al. 2003), causing an accumulation of TAG (Xu et al. 2005). We also observed a greater abundance of proteins involved in chloroplastic fatty acid biosynthesis (Figure 4 and Figure 5). Thus, the similar phenotype of $\operatorname{tg} d 1$ and autophagy mutants as well as the induction of the chloroplastic lipid biosynthetic pathway in these mutants point to a possible limitation in the lipid trafficking between the ER and the chloroplast in autophagy mutants that may delay chloroplast development. However, we 
400

401

402

403

404

405

406

407

408

409

410

411

412

413

414

415

416

417

418

419

420

421

422

423

424

425

426

427

428

429

430

would note that photosynthetic performance was not affected in $\operatorname{tg} d 1$ mutants (Figure 7B-D). Thus, we conclude that limitations in neither chloroplast lipid import nor MGDG-DGDG content are solely responsible for the developmental delay in autophagy mutants compared to WT.

Lipid turnover plays a role in photomorphogenesis and the contribution of autophagy to lipid homeostasis may be the reason of its role in photomorphogenesis

Autophagy mutants are known to have less TAG and reduced TAG mobilization (Fan et al 2019). We observed that autophagy mutants maintained higher abundance of proteins involved in plastid fatty acid biosynthesis (Figure 4 and Figure 5), which could be indicative of a compensatory response to lower levels of fatty acids. The increase of transcripts for lipid biosynthetic enzymes was also recently observed in autophagy mutants (atg12) of maize subjected to carbon starvation (McLoughlin et al. 2020). Based on the hypothesis that the different levels and profiles of lipids may affect photomorphogenesis process in autophagy mutants, we assessed greening in the $s d p 1$ mutants, which like autophagy mutants have impaired capacity to use TAG, to see if they mimic the slow greening phenotype of autophagy mutants. Our results showed that sdpl mutants also have impaired photomorphogenesis (Figure 7), so it is possible that the autophagy-related slow-greening phenotype we observed is due to a limitation in TAG catabolism (Figure 9). In fact, the $s d p 1$ phenotype was more severe than the one observed in autophagy mutants. $S d p 1$ encodes for a lipase that controls fat storage breakdown (Eastmond 2006), and the $s d p 1$ mutant displays an $80 \%$ reduction in lipid hydrolysis rates compared to WT (Li-Beisson et al. 2013). Although we know autophagy mutants have lower TAG content (Minina et al. 2018; Fan et al. 2019), it is not expected that the autophagy mutants would have such a strong reduction in TAG catabolism as $s d p 1$. Therefore the more severe slow greening phenotype in $s d p 1$ than in autophagy mutants might be expected if TAG availability is the explanation of the phenotype.

Energetic restrictions in autophagy mutants also contribute to the delayed photomorphogenesis

Our results showed that the limitations in TAG catabolism (spdl) have more severe consequences in photomorphogenesis than the limitations in lipid trafficking (tgdl). SDP1 is the major TAG lipase involved in lipid reserve mobilization during seedling establishment 
431 (Eastmond 2006; Thazar-Poulot et al. 2015). The sdpl mutants were isolated as mutants

432 displaying a postgerminative growth arrest when sugars are not provided in the medium

433 (Eastmond 2006). This is because SPD1 is ultimately responsible for sucrose production

434 during seedling establishment. In particular, SDP1 degrades TAG to release fatty acids which

435 are transferred to the peroxisome to be converted into succinate, which is used for the

436 synthesis of carbohydrates (Figure 9). Moreover, the physical interaction between lipid

437 droplets and peroxisomes, and thus SDP1 activity, is tightly regulated by sucrose levels, by

438 way of high sucrose levels reducing this interaction, mediated by actin filaments (Cui et al.

439 2016). Therefore, there is significant evidence that SDP1 plays a role in controlling the

440 energetic status of seedlings and now we can infer that this energetic status is also important

441 for optimal photomorphogenesis.

442 Since our seedlings were grown in the dark for 5 days, it is also possible that induced 443 autophagy was playing a role in our experimental conditions to further support the energetic 444 requirement for the photomorphogenic process. In case of carbon deprivation, lipid droplets 445 (or oil bodies) are degraded into fatty acids to produce energy through their catabolism and a 446 specific type of induced autophagy, macrolipophagy, was shown to mediate this degradation 447 (Fan et al. 2019). In this case, the lipid droplets are degraded in the vacuole rather than in the 448 peroxisomes (Figure 9). Thus, it would be expected that SPD1 metabolism is induced 449 concomitantly with induced-autophagy and both pathways contribute cellular resources and 450 energy to sustain photomorphogenesis. To test if energetic restriction in autophagy mutants contributes to the delay in photomorphogenesis we repeated the characterization of autophagy mutants under lower and higher levels of C. Our results confirmed that lower levels of $\mathrm{C}$ exacerbate the greening phenotype of autophagy mutants whereas much higher levels of sucrose can ameliorate them (Figure 8). Therefore, it is possible that the lower capacity of atg seedlings to produce sugars through lipophagy, results in lower capacity to produce ATP to sustain protein biosynthesis (Figure 9).

Very recently, mitophagy was shown to take place in cotyledons during de-etiolation (6h post illumination) and an analogous delay in greening capacity of atg5 was presented (Ma et al. 2021), suggesting that autophagy plays a role in adjusting the number of mitochondria during photomorphogenesis and proposing cross-talk with delayed plastid biogenesis. We show here that the atg greening phenotype is not specific to atg5 and is not constitutive, but conditional on $\mathrm{C}$ status. In addition, we observed that respiration rate per se is not affected in atg mutants 
463

464

465

466

467

468

469

470

471

472

473

474

475

476

477

478

479

480

481

482

483

484

485

486

487

488

489

490

491

492

compared to WT during de-etiolation at least at 12 hours (Figure S4), suggesting that the possible changes in mitochondrial turnover do not affect the overall respiration rate and that mitophagy is unlikely to be the primary process determining the slow greening phenotype. However, the phenotype is likely to relate to the function of both organelles in the context of sugar availability and energetic supply to biosynthetic processes.

Sugars not only play an energetic role but also act as signalling molecule in plants (BaenaGonzález et al. 2007). For instance, sucrose promotes the accumulation of trehalose-6phosphate (T6P) which inhibits Snf1-related kinase 1 (SnRK1), an antagonist of target of rapamycin (TOR), capable of repressing biosynthetic processes and plant growth (BaenaGonzález et al. 2007; Ghillebert et al. 2011). On the other hand, TOR complex promotes metabolic activity and growth, ribosome biogenesis, protein synthesis, and influence the rate of respiration with different substrates (Lastdrager et al. 2014, O'Leary et al 2020). In the first hours of Arabidopsis seedlings transitioning from dark to light, TOR has been shown to be responsible for the phosphorylation of ribosomal protein S6 (RPS6) to enhance protein translation to establish the photosynthetic apparatus (Chen et al. 2018). Therefore, it is possible that the suppression of lipophagy in atg lines, induces a starvation-like condition in atg etiolated seedlings, that induces SnRK1 activity, which in turns phosphorylate and inactivate TOR reducing the translation of the photosynthetic machinery (Figure 9). This putative process would implicate an important role of autophagy and lipophagy in seedling establishment and deserves further exploration.

\section{Conclusion}

We conclude that autophagy influences the rate of etioplast to chloroplast conversion and plays a role in photomorphogenesis most probably by contributing to lipid mobilization and energy production (Figure 9). The effect could be both; indirectly through the modulation of cellular processes that contribute to cellular resources for growth and development, and also directly by etioplasts maintaining higher levels of lipid synthesis machinery to provide TAG for thylakoid membranes and limiting the availability of reductant and ATP inside the plastid for use in the biosynthetic demands of protein import, protein synthesis and photosynthetic protein complex assembly. 
bioRxiv preprint doi: https:/doi org/10.1101/2021.03 25.437007. this version posted March 25. 2021. The copyriaht holder for this preprint (which was not certified by peer review) is the author/funder, who has granted bioRxiv a license to display the preprint in perpetuity. It is made available under aCC-BY-NC-ND 4.0 International license.

494 
495

496

497

498

499

500

501

502

503

504

505

506

507

508

509

510

511

512

513

514

515

516

517

518

519

520

521

522

523

524

525

526

527

\section{Materials and Methods}

\section{Plant materials and growth conditions}

We used arabidopsis (Arabidopsis thaliana) seeds of Col-0 as WT, four autophagic mutants atg2-1 (SALK_076727, Yoshimoto et al. 2009), atg5-1 (SAIL_129B07, Chen et al. 2015), atg7-2 (GABI_655B06, Lai et al. 2011) and atg9-2 (SALK_130796, Zhuang et al. 2017) which we referred as $\operatorname{atg} 2$, atg5, atg7 and $\operatorname{atg} 9$ respectively, a lipid thylakoid permease mutant $\operatorname{tg} d 1$ (Xu et al. 2003), a TAG lipase mutant (sugar-dependent 1-4) sdp1-4 (Eastmond 2006; Fan et al. 2017) and the respective lipid and autophagy double mutants tgdlatg2-1, tgd1 atg5-1, sdp1atg2-1, and sdp1 atg5-1 (Fan et al. 2019).

Growth conditions vary between the developmental transition studied. Three developmental transitions were established; during early seedling development (T1), dark to light greening of seedlings (T2) and light to dark senescence of leaves (T3). For T1 and T2, seeds were surface-sterilized and carefully dispensed on a stainless-steel wire mesh platform (mesh size $1 \mathrm{~mm} ; 3 \mathrm{~cm} \times 3 \mathrm{~cm} \times 3 \mathrm{~cm}$ ) layered previously with $1 \%$ sterilized agarose in a round plastic vessel containing $300 \mathrm{~mL}$ of liquid medium (1/4- strength Murashige and Skoog -MSmedium without vitamins, 1/4- strength Gamborg B5 vitamins solution, 2 mM MES, 1\% [w/v] sucrose, $\mathrm{pH}$ 5.8). For T1, plants were grown under a $16 / 8 \mathrm{~h}$ light/dark period with a light intensity of $100-125 \mu \mathrm{mol} \cdot \mathrm{m}^{-2} \cdot \mathrm{s}^{-1}$ at $22^{\circ} \mathrm{C}$ and samples were collected two days after germination (T1_0d) and then one, three and five days after (T1_1d, T1_3d and T1_5d). For T2, seeds were grown five days in dark (T2_0d), and then grown under 16/8 h light/dark period with a light intensity of $100-125 \mu \mathrm{mol} \cdot \mathrm{m}^{-2} \cdot \mathrm{s}^{-1}$ at $22^{\circ} \mathrm{C}$ for one, three and five days (T2_1d, T2_3d, T2_5d).

For T3, plants were grown in Hoagland's solution and after 21 days (T3_0d_UC), leaves were covered (leaf number three, five, and seven) (C) by aluminum foil to study darkinduced senescence. Covered leaves (leaf number: three, five, seven) and uncovered (UC) leaves (leaf number: four, six, eight) were collected over time course (1 day, 3 days and 5 days) (Uncovered leaves: T3_1d_UC, T3_3dU_C, T3_5d_UC, Covered leaves: T3_1d_C, T3_3d_C, T3_5d_C).

For successive physiological study of the autophagy mutants and all the study of the lipid metabolism mutants, the growth conditions were the same but they were sown on plates containing $0.5 \mathrm{X}$ MS medium supplemented with $0.5 \%$ sucrose (unless otherwise stated) and $0.8 \%$ plant agar. For this, seeds were surface sterilized by 1 min of $70 \%$ ethanol, rinsed once 
with sterile water, 3 min with $20 \%$ sodium hypochlorite, and washed five times with sterile water.

\section{Protein isolation and sample preparation}

Approximately $200 \mathrm{mg}$ of frozen tissue were ground under liquid nitrogen before extraction in $400 \mu \mathrm{L}$ of $125 \mathrm{~mm}$ Tris- $\mathrm{HCl} \mathrm{pH} 7.5,7 \%$ (w/v) SDS, $10 \%$ (v/v) $\beta$-mercaptoethanol, $0.5 \%$ (w/v) PVP40 with Roche protease inhibitor cocktail added at 1 tablet per $50 \mathrm{~mL}$ of extraction buffer. Protein extraction was carried out by chloroform/methanol extraction (Wessel and Flügge 1984) before washing the pellet twice in $80 \%$ (v/v) acetone.

Samples were resuspended in freshly prepared buffer (7M Urea, 2M Thiourea, 50mM $\mathrm{NH}_{4} \mathrm{HCO}_{3}$, and $10 \mathrm{mM}$ DTT) to obtain $15-25 \mu \mathrm{g} / \mu \mathrm{L}$ range after resuspension. Protein concentration was determined by Bradford assay and spectrophotometric measurement at a wavelength of $595 \mathrm{~nm}$ using bovine serum albumin as a standard.

$200 \mu \mathrm{g}$ of protein were treated with $25 \mathrm{mM}$ Iodoacetamide for $30 \mathrm{~min}$ in the dark. The sample solutions were then diluted to below $1 \mathrm{M}$ urea with $50 \mathrm{mM} \mathrm{NH} \mathrm{HCO}_{3}$. For protein digestion, $10 \mathrm{mg}$ of trypsin (dissolved in $0.01 \%[\mathrm{v} / \mathrm{v}]$ trifluoroacetic acid to a concentration of 1 $\mathrm{mg} / \mathrm{mL}$ ) was added to each sample and incubated at $37^{\circ} \mathrm{C}$ overnight. The samples were acidified to $1 \%$ (v/v) with formic acid and solid-phase extraction cleaned using Silica C18 Macrospin columns (The Nest Group). After each of the following steps, solid-phase extraction columns were centrifuged for $3 \mathrm{~min}$ at $150 \mathrm{~g}$ at room temperature. Before loading, the sample columns were washed with $750 \mu \mathrm{L}$ of $70 \%(\mathrm{v} / \mathrm{v})$ acetonitrile, $0.1 \%(\mathrm{v} / \mathrm{v})$ formic acid and charged with $750 \mu \mathrm{L}$ of $5 \%(\mathrm{v} / \mathrm{v})$ acetonitrile, $0.1 \%(\mathrm{v} / \mathrm{v})$ formic acid. After loading the samples onto the columns, two washes with $750 \mu \mathrm{L}$ of $5 \%(\mathrm{v} / \mathrm{v})$ acetonitrile, $0.1 \%(\mathrm{v} / \mathrm{v})$ formic acid were carried out, followed by two elution steps with $750 \mu \mathrm{L}$ of $70 \%(\mathrm{v} / \mathrm{v})$ acetonitrile, $0.1 \%(\mathrm{v} / \mathrm{v})$ formic acid. The eluate was dried under vacuum and resuspended in $5 \%(\mathrm{v} / \mathrm{v})$ acetonitrile, $0.1 \%(\mathrm{v} / \mathrm{v})$ formic acid to a final concentration of $1 \mu \mathrm{g} / \mu \mathrm{L}$ for MS.

\section{LC-MS/MS for dynamic MRM analysis}

Using an Agilent 1290 Infinity II LC system, $10 \mu \mathrm{g}$ of each sample were loaded onto an Agilent AdvanceBio Peptide Map column (2.1 x 250mm 2.7-Micron, P.N. 651750- 902), which was heated to $60{ }^{\circ} \mathrm{C}$. Peptides were eluted over a 30 min gradient $(0-15 \min 3 \%$ [v/v] acetonitrile $0.1 \%$ [v/v] formic acid to $45 \%$ [v/v] acetonitrile $0.1 \%$ [v/v] formic acid; $15-15.5$ $\min 45 \%[\mathrm{v} / \mathrm{v}]$ acetonitrile $0.1 \%[\mathrm{v} / \mathrm{v}]$ formic acid to $100 \%[\mathrm{v} / \mathrm{v}]$ acetonitrile $0.1 \%[\mathrm{v} / \mathrm{v}]$ formic acid; $15.5-16 \min 100 \%[\mathrm{v} / \mathrm{v}]$ acetonitrile $0.1 \%[\mathrm{v} / \mathrm{v}]$ formic acid to $3 \%[\mathrm{v} / \mathrm{v}]$ 
acetonitrile $0.1 \%[\mathrm{v} / \mathrm{v}]$ formic acid; $16-30 \min 3 \%[\mathrm{v} / \mathrm{v}]$ acetonitrile $0.1 \%[\mathrm{v} / \mathrm{v}]$ formic acid) directly into the Agilent 6495 Triple Quadrupole MS for detection.

\section{Analysis of targeted MRM data}

566 Before conducting MRM analysis for the individual samples, a preliminary MRM analysis was performed using pooled samples $(n=4)$ of all growth and developmental stages to obtain transition information, such as detectability in tissues and suitability of transition. For organelle-specific assay, 680 peptides (2231 transitions) representing 76 proteins were targeted. After obtaining the resulting MRM data, the final target list was selected using the following criteria: at least one peptide was selected per protein, and at least three transitions (one quantifier and two qualifiers) per peptide were chosen. Individual MRM analysis was performed using 221 transitions, corresponding to 31 proteins.

Raw data files from MRM analysis were processed using Skyline. Peak area integration was confirmed manually to correct potentially wrongly assigned targets. For further analysis, the integrated area of the quantifier ion was used. Normalization was performed to correct for differences between sample runs. Therefore, every peptide within a sample was divided by the median of all detected peptides in that sample. Then, to give peptides the same weight, normalized data were scaled by dividing each peptide by the median of that peptide across all samples.

\section{$L C-M S / M S$ for shotgun proteomics}

583 Digested peptides were resuspended in $20 \mu \mathrm{L}$ of $2 \%$ acetonitrile (v/v), $0.1 \%$ formic acid $584(\mathrm{v} / \mathrm{v})$, and $5 \mu \mathrm{l}$ of this resuspension was loaded onto a C18 high capacity nano-LC chip 585 (Agilent) in 98\% Buffer A $(0.1 \%$ (v/v) formic acid in Optima grade water (Fisher)) and 2\%

586 Buffer B (0.1\% formic acid in Optima grade acetonitrile (Fisher)) using a 1200 series capillary pump (Agilent). Following loading, samples were eluted from the C18 column and into an inline 6550 Series QTOF mass spectrometer (Agilent) with a 1200 series nano pump (Agilent) using the following gradient: $2 \% \mathrm{~B}$ to $45 \% \mathrm{~B}$ in 26 minutes, $45 \%$ to $60 \% \mathrm{~B}$ in 3 minutes, $60 \%$ to $100 \% \mathrm{~B}$ in 1 minute. The samples were analyzed in sequence, and a blank was run between samples. The QTOF was operated in a data- dependent fashion with an MS spectrum collected prior to the three most abundant ions subjected to tandem mass spectrometry from doubly, triply, and higher charge states. Ions were dynamically excluded for 0.4 minutes following fragmentation. MS data were collected at eight spectra per second, 
595

596

597

598

599

600

601

602

603

604

605

606

607

608

609

610

611

612

613

614

615

616

617

618

619

620

621

622

623

624

625

626

627

while MS/MS spectra were collected at three spectra per second with a minimum threshold of 10,000 counts and a target of 25,000 ions per MS/MS event.

\section{Label-Free Quantitative shotgun proteomics data analysis}

Raw data files were analyzed by the MaxQuant quantitative proteomics software package (Cox and Mann 2008). Peak lists were searched against the Arabidopsis Fasta database TAIR10_pep_20101214. Each genotype was represented by four biological replicates in three different times, $0 \mathrm{~h}, 12 \mathrm{~h}$ and $24 \mathrm{~h}$. Statistical analysis of Label-Free Quantitation (LFQ) values from MaxQuant was performed using the Perseus software (http://www.perseusframework.org/) from MaxQuant (Tyanova et al. 2016). Proteins were filtered according to the presence in at least $70 \%$ of the samples tested and being annotated as plastid proteins according to SUBA4 (https://suba.live) consensus column. Subsequently, differential enrichment analysis between autophagy mutants and WT plants was performed and additional filtering was applied to retain those proteins that were differentially enriched in at least three out of four atg mutants and in at least two out of three times tested. Multiplesample ANOVA test was used to determine significance. A protein was considered differentially expressed if the permutation-based false discovery rate (FDR) adjusted p-values were below 0.05 . The resulting dataset was hierarchically clustered to produce heatmaps using the Z-score value in Perseus (Tyanova et al. 2016). Additionally, a GO term and KEGG pathways enrichments analysis was performed using Perseus (Tyanova et al. 2016). The mass spectrometry proteomics data have been deposited to the ProteomeXchange Consortium via the PRIDE partner repository with the dataset identifier PXD024878.

\section{RNA isolation and quality assessment}

Arabidopsis total RNA was isolated using a CTAB-based extraction method (ArmaregoMarriott et al. 2019) with modifications that are detailed on protocols.io (dx.doi.org/10.17504/protocols.io.3f6gjre). The protocol described here was an effective alternative to TRIzol-based extractions for recovering RNA from juvenile de- etiolated tissues. Plant tissues were frozen and ground in liquid nitrogen using mortar and pestle to obtain a fine powder. Ground tissue was kept in safe-lock tubes in liquid nitrogen (or returned to $-80 \mathrm{C}$ storage) until all samples were processed. Pre-warmed $\left(65^{\circ} \mathrm{C}\right)$ extraction buffer $\quad(1 \mathrm{~mL}) \quad[2 \% \quad$ Hexadecyltrimethylammonium bromide $\quad$ (CTAB), 2\% polyvinylpyrrolidone K 30, $100 \mathrm{~mm}$ 2-amino-2-(hydroxymethyl)-1,3-propanediol (TRIS)- 
$\mathrm{HCl}, \mathrm{pH}$ 8.0, $25 \mathrm{~mm}$ EDTA, $2.0 \mathrm{~m} \mathrm{NaCl}, 0.5 \mathrm{~g}$ 1-1 spermidine, 2\% 2- mercaptoethanol] was added to each tube, mixed well and incubated for $5 \mathrm{mins}$ at $65^{\circ} \mathrm{C}$. Then $200 \mu \mathrm{l}$ of chloroform:IAA (24:1) was added, and spun at 14,000 rcf for 10 mins. The upper aqueous phase was recovered. Later an equal volume of $5 \mathrm{M} \mathrm{LiCl}$ was added into the aqueous layer and mixed well. After overnight incubation at $-20^{\circ} \mathrm{C}$, the RNA pellet was collected by centrifugation at $14000 \mathrm{rcf}$ for $20 \mathrm{~min}$ at $4^{\circ} \mathrm{C}$. Then the pellet was washed with $80 \%$ ethanol and dissolved in $200 \mu \mathrm{l}$ RNase-free water. The quality of RNA samples was assessed with BioAnalyser / LabChip GXII or by visualization of 50 ng RNA on a 1\% agarose gel. RNA was treated with TURBO DNase (Ambion/ThermoFisher) followed by AMPure cleanup (detailed protocol is available in GitHub repository https://goo.gl/jMXW1F). RNA was quality assessed for RIN scores using a Bioanalyser (Agilent) or a GXII labchip (Perkin Elmer). RNA quantity and purity was assessed using the ND-1000 spectrophotometer (NanoDrop Technologies). RNA quality was assessed using the LabChip GXII (Perkin-Elmer).

641

\section{Library preparation and sequencing}

643 For total RNA-seq, 1 ug of RNA was depleted for rRNA using the Illumina Plant Ribo-Zero 644 kit, following the manufacturer's protocol (Ribo-Zero Magnetic Kits Guide Rev A and 645 TruSeq Stranded Total RNA Guide Rev E), except all reaction volumes were adjusted by 1/2 646 (Crisp et al. 2017). Depleted RNA was then purified and fragmented (7 min) using the 647 Illumina Truseq mRNA stranded kit with 1/3 adjusted reaction volumes (Crisp et al. 2017). 648 An optimal cycle number of 8 was used for the amplification of the final libraries. Samples 649 were pooled in equal molar ratios and sequenced on the NextSeq500 (75 bp single-end) at the 650 ACRF Biomolecular Research Facility (Australian National University, ACT, Australia). 651 Raw sequence data is available from GEO (GSE156677).

652

\section{Quantification of transcripts}

654 Raw sequencing reads were trimmed to remove adaptor sequences and low-quality base calls 655 (-q 20-length 80-stringency 3) by using Trim Galore! (v0.4.1) (Cheng et al. 2016). Then 656 Subsequent read quality was assessed using FastQC (v0.10.1) (Andrews, 2010). Transcript 657 quantification was performed using Kallisto (Bray et al. 2016) in conjunction with the 658 Arabidopsis thaliana Reference Transcript Dataset 2 (AtRTD2_19April2016.gtf), which 659 contains a comprehensive set of transcript sequences (Cao et al. 2017). Code is available on 660 GitHub (https://github.com/dtrain16/NGS-scripts/blob/master/RNA/RNAseq_kallisto_v1.sh). 
Green Index determination

663 To semi quantitatively assess the greening of seedling during the light to dark transition, 664 photos of the different genotypes were taken and independent cotyledons $(n=10)$ for each 665 genotype and time point were used to determine the Green Index (GI). Moreover, the GI determinations were performed in five independent experiments. GI was performed by recording the RGB values obtained from as the average of a 5x5 pixel area using Affinity Photo software. Then GI was calculated as follows:

669

$673 \mathrm{R}, \mathrm{G}$, and $\mathrm{B}$ refers to the red, green and blue (R, G, B) of the RGB values and ranges from 0 674 to 255 .

675

\section{Correlation analysis between transcriptomic and proteomic data}

677 A correlation between proteomic and transcriptomic data was performed for the subset of 678 plastid proteins IDs that matched the criteria of being differentially enriched in at least three 679 out of four autophagy mutants tested and consistent in at least two out of three time point 680 tested. To do this, the Log2 LFQ intensities were normalized to the average for this subset of 681 proteins and the equivalent normalization of Log2 TPM was done for the transcriptomic data. 682 The normalized Log2 LFQ and Log2 TPM were plotted as scatter plot in R and a Pearson 683 correlation test was performed in Excel to determine the Person correlation coefficient, the $p$ 684 value and its biological significance.

\section{Chlorophyll fluorescence imaging and photosynthetic parameters}

687 Images of chlorophyll fluorescence were measured using the MAXI-Imaging PAM 688 fluorometer system (Walz, Germany) (Barbagallo et al. 2003). Plants were dark-adapted for $68915 \mathrm{~min}$ before minimal fluorescence (Fo) was measured. Then, maximal fluorescence (Fm) 690 was measured during $800 \mathrm{~ms}$ exposure to a saturating pulse, having a photon flux density 691 (PFD) of $4800 \mu \mathrm{mol} \cdot \mathrm{m}^{-2} \cdot \mathrm{s}^{-1}$. Thereafter, Fv/Fm, the maximum quantum efficiency of PSII 692 photochemistry, was calculated. Electron transport rate (ETR) parameter was determined 693 immediately after Fo and Fm determination. Twenty measurements were used to describe the 
694 light curve for ETR, from 0 to 1076 photosynthetic active radiation (PAR), with a 20 seconds gap between measurements. At least six biological replicates were used per experiment and four independent experiments were combined to obtain the final result.

697

698

699

700

701

702

703

704

705

706

707

708

709

710

711

712

713

714

715

716

717

718

719

720

721

722

723

724

725

726

\section{Chlorophyll extraction and analysis}

Frozen de-etiolating seedlings were ground in a mortar under liquid nitrogen. $20 \mathrm{mg}$ of tissue were transferred into $400 \mu \mathrm{L}$ of methanol and shaken for two minutes at $30 \mathrm{hz}$. Samples were centrifuged for two minutes at $14000 \mathrm{~g}$ at $4^{\circ} \mathrm{C}$. The supernatant was re-extracted with a second aliquot of $400 \mu \mathrm{L}$ of methanol, shaken for 2 minutes, and debris pelleted before measurement at $652 \mathrm{~nm}$ and $665 \mathrm{~nm}$. The formula used to determine the chlorophyll content were: chlorophyll a $(\mu \mathrm{g} / \mathrm{mL})=(-8.0962 \mathrm{~A} 652,1 \mathrm{~cm}+16.5169$ A665, $1 \mathrm{~cm})$, chlorophyll b $(\mu \mathrm{g} / \mathrm{mL})=(27.4405 \mathrm{~A} 652,1 \mathrm{~cm}-12.1688 \mathrm{~A} 665,1 \mathrm{~cm})($ Warren 2008).

\section{Microscopic analysis of chloroplast}

Confocal laser scanning microscopy (CLSM) (Nikon A1-Si Confocal Microscope) was used to examine plastids during the photomorphogenesis transition. Chlorophyll auto-fluorescence was used to visualize with filters set at excitation: $488 \mathrm{~nm}$; emission: $640 \mathrm{~nm}$. Images were acquired sequentially or simultaneously where appropriate (Kodama 2016). Resulting regions of interest (ROIs) were selected as $1024 \times 1024 \mathrm{px}^{2}$; area $\left(\right.$ in $\mathrm{px}^{2}$ ) where a pixel resolution size $0.07 \mu \mathrm{m} / \mathrm{px}$. A total of 5 biological replicates were used to determine the chloroplast size and 10 biological replicates for counting the chloroplasts.

\section{Liquid-phase oxygen evolution}

Oxygen evolution was determined using a Clark type polarographic oxygen electrode (Oxygraph Plus System). Oxygen electrode were assembled, stabilized for $1 \mathrm{~h}$ and calibrated every day prior measurements. The liquid phase consisted of $1 \mathrm{mM} \mathrm{NaHCO}, 10 \mathrm{mM}$ HEPES-KOH, pH 7.5 buffer, which was freshly prepared every day. Seedlings were weighed on an analytical scale and fresh weight (FW) was recorded. A minimum of $6 \mathrm{mg}$ of seedlings was used in every case. Oxygraph temperature was set to $25^{\circ} \mathrm{C}$ and stirrer speed at $70 \mathrm{rpm}$. Seedlings were transferred to the Oxygraph chamber containing $2 \mathrm{~mL}$ of the abovementioned buffer, and three phase measurements were taken. The first phase consisted of 3-5 min of measurement under dark condition to determine oxygen consumption (i.e. respiration). After having at least 3 min of linear slope, the second phase was initiated by 
turning on the light and the oxygen evolution was recorded for at least 5 additional min. A 12V 100W Olympus LG-PS2 Fibre Optic Illuminator was used as source of light to induce photosynthetic activity. After this time, light was turned off again and another 3-5 min were recorded in darkness (third phase). Respiration was calculated as the average of the oxygen consumption determined from the slope of phase 1 and 3 per FW of sample and expressed as nmol $\mathrm{O}_{2} \cdot \min ^{-1} \cdot \mathrm{g}^{-1} \mathrm{FW}$. Light dependent oxygen production (i.e. photosynthesis) was calculated as the difference between the slope obtained in light condition (phase 2) and darkness (phase 1 and 3), and expressed as $\mathrm{nmol} \mathrm{O}_{2} \cdot \mathrm{min}^{-1} \cdot \mathrm{g}^{-1} \mathrm{FW}$. The ratio between these two parameters (photosynthesis/respiration) was also determined to give an indication how photoautotrophic the seedlings were and thus their developmental stage. Values higher than 1 mean the plant produce more oxygen than what it consumes under light condition indicating a more developed state, whereas values lower than 1 indicates the plant consume more oxygen than what it produces indicating a less developed state. The measurements consisted of at least six biological replicates, each biological replicate consisting of multiple seedlings.

\section{Supplemental Data}

Supplemental Table 1. Green Index Tukey's HSD comparison for autophagy and lipid metabolism mutants and WT.

Figure S1. Abundance of plastid MRM protein biomarkers in WT during germination (T1) and light to dark transition (T3).

Figure S2. Organelles specific protein abundance profiling of WT and autophagy mutants in the dark to light transition

Figure S3. Comparison of relative apparent electron transport rate (ETR) plots between the different genotypes and WT.

Figure S4. Liquid-phase respiration and photosynthetic activity in different genotypes and WT.

Figure S5. Comparison of relative apparent electron transport rate (ETR) plots between the different genotypes and WT

Figure S6. Comparison of relative apparent electron transport rate (ETR) plots for WT and 


\section{Acknowledgment}

We thank the authors referenced in methods for provision of autophagy mutants and especially thank Dr Changcheng Xu from Brookhaven National Laboratory for provision of the series of $s d p 1$, tgdl and atg double mutants reported in Fan et al. (2019) that were used in this study. Dr Brendan O'Leary (The University of Western Australia) is thanked for critical reading of the article. AWY was supported by a UWA Research Training Program International Student Scholarship. This work was support by Australian Research Council funding to AHM and BJP (CE140100008). We acknowledge the Biomolecular Resource Facility at the ANU for performing Illumina sequencing and the provision of computational infrastructure by the National Computational Infrastructure supported under the National

\section{Author contributions}

AWY and SS performed most of the experiments and data analysis, RF and OD assisted with mass spectrometry and data analysis, DRG and BJP assisted with RNAseq and data analysis, AHM, OD, LL, ES and SS designed the experiments. SS and AHM wrote the paper and all authors contributed to revising it.

\section{REFERENCES}

Armarego-Marriott, Kowalewska, Burgos, Fischer, Thiele, Erban, Strand, Kahlau, Hertle, Kopka, et al (2019) Highly resolved systems biology to dissect the etioplast-tochloroplast transition in tobacco leaves. Plant Physiol 180:654-681

Avin-Wittenberg (2018) Autophagy and its Role in Plant Abiotic Stress Management. Plant Cell Environ. https://doi.org/10.1111/pce.13404

Avin-Wittenberg, Bajdzienko, Wittenberg, Alseekh, Tohge, Bock, Giavalisco, Fernie (2015) in Arabidopsis seedlings under carbon starvation. Plant Cell 27:306-322

Awai, Xu, Tamot, Benning (2006) A phosphatidic acid-binding protein of the chloroplast inner envelope membrane involved in lipid trafficking. Proc Natl Acad Sci U S A 
Baena-González, Rolland, Thevelein, Sheen (2007) A central integrator of transcription networks in plant stress and energy signalling. Nature 448:938-942

Barbagallo, Oxborough, Pallett, Baker (2003) Rapid, noninvasive screening for perturbations of metabolism and plant growth using chlorophyll fluorescence imaging. Plant Physiol $132: 483-493$

Bassham (2007) Plant autophagy - more than a starvation response. Curr Opin Plant Biol 10:587-593

Bray, Pimentel, Melsted, Pachter (2016) Near-optimal probabilistic RNA-seq quantification. Nat Biotechnol 34:525-527

815 Broda, Millar, Van Aken (2018) Mitophagy: A Mechanism for Plant Growth and Survival. Trends Plant Sci 23:434-450

817 Cai, Li, Liu, Wang, Zhou, Xu, Xiong (2017) COP1 integrates light signals to ROP2 for cell cycle activation. Plant Signal Behav 12:

819 Calixto, Guo, James, Tzioutziou, Entizne, Panter, Knight, Nimmo, Zhang, Brown (2018) Rapid and dynamic alternative splicing impacts the arabidopsis cold response transcriptome[CC-BY]. Plant Cell 30:1424-1444

Cao, Ni, Zhang, Shi, Xu, Yan, Zhang (2017) Alterations in the proteome of wheat primary roots after wortmannin application during seed germination. Acta Physiol Plant 39:223

Chen, Liao, Qi, Xie, Huang, Tan, Zhai, Yuan, Zhou, Yu, et al (2015) Autophagy contributes to regulation of the hypoxia response during submergence in Arabidopsis thaliana. Autophagy 11:2233-2246

Chen, Liu, Xiong, Sheen, Wu (2018) TOR and RPS6 transmit light signals to enhance protein translation in deetiolating Arabidopsis seedlings. Proc Natl Acad Sci U S A 115:1282312828

Cheng, Teo, Krueger, Rock, Koh, Choi, Vogel (2016) Differential dynamics of the mammalian mRNA and protein expression response to misfolding stress. Mol Syst Biol $12: 855$

Chung, Suttangkakul, Vierstra (2009) The ATG autophagic conjugation system in maize: ATG transcripts and abundance of the ATG8-lipid adduct are regulated by development and nutrient availability. Plant Physiol 149:220-234

836 Cox, Mann (2008) MaxQuant enables high peptide identification rates, individualized p.p.b.range mass accuracies and proteome-wide protein quantification. Nat Biotechnol 
Crisp, Ganguly, Smith, Murray, Estavillo, Searle, Ford, Bogdanović, Lister, Borevitz, et al (2017) Rapid recovery gene downregulation during excess-light stress and recovery in arabidopsis. Plant Cell 29:1836-1863

Cui, Hayashi, Otomo, Mano, Oikawa, Hayashi, Nishimura (2016) Sucrose production mediated by lipid metabolism suppresses the physical interaction of peroxisomes and oil bodies during germination of arabidopsis thaliana. J Biol Chem 291:19734-19745

Di Berardino, Marmagne, Berger, Yoshimoto, Cueff, Chardon, Masclaux-Daubresse, Reisdorf-Cren (2018) Autophagy controls resource allocation and protein storage accumulation in Arabidopsis seeds. J Exp Bot 69:1403-1414

Eastmond (2006) Sugar-dependent1 encodes a patatin domain triacylglycerol lipase that initiates storage oil breakdown in germinating Arabidopsis seeds. Plant Cell 18:665-675

Fan, $\mathrm{Yu}, \mathrm{Xu}$ (2017) A central role for triacylglycerol in membrane lipid breakdown, fatty acid $\beta$-oxidation, and plant survival under extended darkness. Plant Physiol 174:15171530

Fan, Yu, Xu (2019) Dual role for autophagy in lipid metabolismin arabidopsis. Plant Cell $31: 1598-1613$

Ghillebert, Swinnen, Wen, Vandesteene, Ramon, Norga, Rolland, Winderickx (2011) The AMPK/SNF1/SnRK1 fuel gauge and energy regulator: Structure, function and regulation. FEBS J 278:3978-3990

Guiboileau, Yoshimoto, Soulay, Bataillé, Avice, Masclaux-Daubresse (2012) Autophagy machinery controls nitrogen remobilization at the whole-plant level under both limiting and ample nitrate conditions in Arabidopsis. New Phytol 194:732-740

Guo, Tzioutziou, Stephen, Milne, Calixto, Waugh, Brown, Zhang (2020) 3D RNA-seq: a powerful and flexible tool for rapid and accurate differential expression and alternative splicing analysis of RNA-seq data for biologists. RNA Biol. https://doi.org/10.1080/15476286.2020.1858253

Havé, Luo, Tellier, Balliau, Cueff, Chardon, Zivy, Rajjou, Cacas, Masclaux-Daubresse (2019) Proteomic and lipidomic analyses of the Arabidopsis atg5 autophagy mutant reveal major changes in endoplasmic reticulum and peroxisome metabolisms and in lipid composition. New Phytol 223:1461-1477

Heredia-Martínez, Andrés-Garrido, Martínez-Force, Pérez-Pérez, Crespo (2018) Chloroplast damage induced by the inhibition of fatty acid synthesis triggers autophagy in chlamydomonas. Plant Physiol 178:1112-1129

Hooper, Stevens, Saukkonen, Castleden, Singh, Mann, Fabre, Ito, Deery, Lilley, et al (2017) 
873

874

875

876

877

878

879

880

881

882

883

884

885

886

887

888

889

890

891

892

893

894

895

896

897

898

899

900

901

902

903

904

905

906

Multiple marker abundance profiling: combining selected reaction monitoring and datadependent acquisition for rapid estimation of organelle abundance in subcellular samples. Plant J 92:1202-1217

Ishida, Izumi, Wada, Makino (2014) Roles of autophagy in chloroplast recycling. Biochim Biophys Acta - Bioenerg 1837:512-521

Izumi, Ishida, Nakamura, Hidema (2017) Entire Photodamaged Chloroplasts Are Transported to the Central Vacuole by Autophagy. Plant Cell 29:377-394

Izumi, Wada, Makino, Ishida (2010) The autophagic degradation of chloroplasts via rubiscocontaining bodies is specifically linked to leaf carbon status but not nitrogen status in arabidopsis. Plant Physiol 154:1196-1209

Janse van Rensburg, Van den Ende, Signorelli (2019) Autophagy in plants: Both a puppet and a puppet master of sugars. Front Plant Sci 10:

Jiang, Zhu, Wang, Hou (2020) Autophagy-related 2 regulates chlorophyll degradation under abiotic stress conditions in arabidopsis. Int J Mol Sci 21:4515

Kodama (2016) Time gating of chloroplast autofluorescence allows clearer fluorescence imaging in planta. PLoS One 11:e0152484

LaBrant, Barnes, Roston (2018) Lipid transport required to make lipids of photosynthetic membranes. Photosynth Res 138:345-360

Lai, Wang, Zheng, Fan, Chen (2011) A critical role of autophagy in plant resistance to necrotrophic fungal pathogens. Plant J 66:953-968

Lastdrager, Hanson, Smeekens (2014) Sugar signals and the control of plant growth and development. J Exp Bot 65:799-807

Law, Narsai, Whelan (2014) Mitochondrial biogenesis in plants during seed germination. Mitochondrion 19:214-221

Li-Beisson, Shorrosh, Beisson, Andersson, Arondel, Bates, Baud, Bird, DeBono, Durrett, et al (2013) Acyl-Lipid Metabolism. In: The Arabidopsis Book. p e0161

Li, Chung, Vierstra (2014) AUTOPHAGY-RELATED11 plays a critical role in general autophagy- and senescence-induced mitophagy in Arabidopsis. Plant Cell 26:788-807

Li, Gao, Benning, Sharkey (2012) Characterization of photosynthesis in Arabidopsis ER-toplastid lipid trafficking mutants. Photosynth Res 112:49-61

Li, Yu (2018) Chloroplast galactolipids: The link between photosynthesis, chloroplast shape, jasmonates, phosphate starvation and freezing tolerance. Plant Cell Physiol 59:11281134

Luo, Zhou, Masclaux-Daubresse, Wang, Wang, Zheng (2019) Morphological and 
physiological responses to contrasting nitrogen regimes in Populus cathayana is linked to resources allocation and carbon/nitrogen partition. Environ Exp Bot 162:247-255

Ma, Liang, Zhao, Wang, Ma, Mai, Andrade, Zeng, Grujic, Jiang, et al (2021) Article Friendly mediates membrane depolarization- induced mitophagy in Arabidopsis. Curr Biol doi.org/10.1016/j.cub.2021.02.034.

912 Marshall, Vierstra (2018) Autophagy: The Master of Bulk and Selective Recycling. Annu Rev Plant Biol 69:173-208

Masclaux-Daubresse, Chen, Havé (2017) Regulation of nutrient recycling via autophagy. Curr Opin Plant Biol 39:8-17

McLoughlin, Augustine, Marshall, Li, Kirkpatrick, Otegui, Vierstra (2018) Maize multiomics reveal roles for autophagic recycling in proteome remodelling and lipid turnover. Nat Plants 4:1056-1070

McLoughlin, Marshall, Ding, Chatt, Kirkpatrick, Augustine, Li, Otegui, Vierstra (2020)

Michaud, Jouhet (2019) Lipid trafficking at membrane contact sites during plant development and stress response. Front Plant Sci 10:1-10

Minina, Moschou, Vetukuri, Sanchez-Vera, Cardoso, Liu, Elander, Dalman, Beganovic, Lindberg Yilmaz, et al (2018) Transcriptional stimulation of rate-limiting components of the autophagic pathway improves plant fitness. J Exp Bot 69:1415-1432

Nukarinen, Ngele, Pedrotti, Wurzinger, Mair, Landgraf, Börnke, Hanson, Teige, BaenaGonzalez, et al (2016) Quantitative phosphoproteomics reveals the role of the AMPK plant ortholog SnRK1 as a metabolic master regulator under energy deprivation. Sci Rep $6: 1-19$

O’Leary, Oh, Lee, Millar (2020). Metabolite regulatory interactions control plant respiratory metabolism via Target of Rapamycin (TOR) Kinase activation. Plant Cell. doi.org/10.1105/tpc.19.00157

Pfeiffer, Janocha, Dong, Medzihradszky, Schöne, Daum, Suzaki, Forner, Langenecker, Rempel, et al (2016) Integration of light and metabolic signals for stem cell activation at the shoot apical meristem. Elife 5:

Pogson, Ganguly, Albrecht-Borth (2015) Insights into chloroplast biogenesis and development. Biochim Biophys Acta - Bioenerg 1847:1017-1024

Ritchie, Phipson, Wu, Hu, Law, Shi, Smyth (2015) Limma powers differential expression analyses for RNA-sequencing and microarray studies. Nucleic Acids Res 43:e47 
941

942

943

944

945

946

947

948

949

950

951

952

953

954

955

956

957

958

959

960

961

962

963

964

965

966

967

968

969

970

971

972

973

974

Robinson, McCarthy, Smyth (2010) edgeR: A Bioconductor package for differential expression analysis of digital gene expression data. Bioinformatics 26:139-140

Robinson, Oshlack (2010) A scaling normalization method for differential expression analysis of RNA-seq data. Genome Biol 11:R25

Rudowska, Gieczewska, Mazur, Garstka, Mostowska (2012) Chloroplast biogenesis Correlation between structure and function. Biochim Biophys Acta - Bioenerg 1817:1380-1387

Schnurr, Shockey, De Boer, Browse (2002) Fatty acid export from the chloroplast. Molecular characterization of a major plastidial acyl-coenzyme A synthetase from Arabidopsis. Plant Physiol 129:1700-1709

Signorelli, Tarkowski, Van den Ende, Bassham (2019) Linking Autophagy to Abiotic and Biotic Stress Responses. Trends Plant Sci 24:

Soneson, Love, Robinson (2016) Differential analyses for RNA-seq: Transcript-level estimates improve gene-level inferences [version 2; referees: 2 approved]. F1000Research 4:1521

Thazar-Poulot, Miquel, Fobis-Loisy, Gaude (2015) Peroxisome extensions deliver the Arabidopsis SDP1 lipase to oil bodies. Proc Natl Acad Sci U S A 112:4158-4163

Tyanova, Temu, Cox (2016) The MaxQuant computational platform for mass spectrometrybased shotgun proteomics. Nat Protoc 11:2301-2319

Warren (2008) Rapid measurement of chlorophylls with a microplate reader. J Plant Nutr $31: 1321-1332$

Waters, Langdale (2009) The making of a chloroplast. EMBO J 28:2861-2873

Wessel, Flügge (1984) A method for the quantitative recovery of protein in dilute solution in the presence of detergents and lipids. Anal Biochem 138:141-143

$\mathrm{Xu}$, Fan, Froehlich, Awai, Benning (2005) Mutation of the TGD1 chloroplast envelope protein affects phosphatidate metabolism in Arabidopsis. Plant Cell 17:3094-3110

$\mathrm{Xu}$, Fan, Riekhof, Froehlich, Benning (2003) A permease-like protein involved in ER to thylakoid lipid transfer in Arabidopsis. EMBO J 22:2370-2379

$\mathrm{Xu}$, Moellering, Muthan, Fan, Benning (2010) Lipid transport mediated by arabidopsis TGD proteins is unidirectional from the endoplasmic reticulum to the plastid. Plant Cell Physiol 51:1019-1028

Yoshimoto, Jikumaru, Kamiya, Kusano, Consonni, Panstruga, Ohsumi, Shirasu (2009) Autophagy negatively regulates cell death by controlling NPR1-dependent salicylic acid signaling during senescence and the innate immune response in Arabidopsis. Plant Cell 
976 Young, Bartel (2016) Pexophagy and peroxisomal protein turnover in plants. Biochim

978 Zhuang, Chung, Cui, Lin, Gao, Kang, Jiang (2017) ATG9 regulates autophagosome

979 progression from the endoplasmic reticulum in Arabidopsis. Proc Natl Acad Sci

980 114:E426-E435

981

982 
984 Figure 1. The dark to light transition for seedling greening is affected in atg lines and

985 characterized by less abundant chloroplast markers. A. Experimental design showing the 986 dark to light transition. B. Abundance of plastid MRM biomarkers, viz. AT1G06680.1 987 (PHOTOSYSTEM II SUBUNIT P-1), AT1G09340.1 (CHLOROPLAST RNA BINDING), 988 AT1G15820.1 (LIGHT HARVESTING COMPLEX B6 OF PHOTOSYSTEM II), and 989 AT1G74470.1 (GERANYLGERANYL DIPHOSPHATE REDUCTASE), during the dark to 990 light transition in WT. The y- axis of the line graph represents averaged peak intensity of 991 three biological samples $(n=3)$ of these markers over the time course. Statistical analyses 992 were performed by one-way ANOVA, followed by Tukey's HSD test compared to the Od 993 time (*P < 0.05 ,). C. Abundance of plastid MRM biomarkers in WT Col-0 and autophagy 994 mutants atg2, atg 5, atg 7 and atg9 during the dark to light transition (0, 1, 3 and 5 days). 995 Means \pm SE for $\mathrm{n}=4$ are shown. Asterisks denote statistically significant differences at the $\mathrm{P}$ 996 $<0.05$ level among treatment groups within a time-point.

Figure 2. The dark to light transition in autophagy mutants is characterized by slower greening, lower chlorophyll content, and reduced number and size of chloroplast than the WT. A. Experimental design to study the dark to light transition for seedling greening. Seeds were germinated and grown on MS solid medium supplemented with $0.5 \%$ sucrose for 5 days in dark and then exposed to light for $0 \mathrm{~h}, 12 \mathrm{~h}$ and $24 \mathrm{~h}$. B. Phenotype of WT and autophagy mutants at $0 \mathrm{~h}, 12 \mathrm{~h}$ and $24 \mathrm{~h}$ of light treatment. C. Green Index for WT and autophagy mutants at $0 \mathrm{~h}, 12 \mathrm{~h}$ and $24 \mathrm{~h}$ of light treatment. The grey regression represents $+/-95 \%$ confidence level intervals for prediction for the 2-order polynomial regression. D. Chlorophyll content for WT and autophagy mutants at $0 \mathrm{~h}, 12 \mathrm{~h}$ and $24 \mathrm{~h}$ of light treatment. ( $\mathrm{n}=4$ independent biological replicates with three technical replicates per group). E. Chlorophyll autofluorescence of WT and autophagy mutants at $12 \mathrm{~h}$ and $24 \mathrm{~h}$ of light treatment. Autofluorescence was detected by CLSM at $640 \mathrm{~nm}$ using a excitation of $488 \mathrm{~nm}$ (scale bars: $20 \mu \mathrm{m})$. F. Number of plastids per area, and G. plastid area for WT and autophagy mutants at $12 \mathrm{~h}$ and $24 \mathrm{~h}$ of light treatment. Resolution size $0.07 \mu \mathrm{m} / \mathrm{px}$, dimension of $1024 \mathrm{x} 1024 \mathrm{px}$ (equivalent to $1.04 \mathrm{MPx}^{2}$ ) of autophagy mutants with control WT are shown $(\mathrm{n}=5$ independent biological replicates per group). Asterisks indicate $\mathrm{P}$ values $\leq 0.05$ by two-way analysis of variance (ANOVA) and Tukey HSD multiple comparison test. Data are

1016 Figure 3. Photosynthetic performance of autophagy mutants during the dark to light 1017 transition. A. $\mathrm{F}_{\mathrm{V}} / \mathrm{F}_{\mathrm{M}}$ images for WT and autophagy mutants at $12 \mathrm{~h}$ and $24 \mathrm{~h}$. B. Analysis of $1018 \mathrm{~F}_{\mathrm{V}} / \mathrm{F}_{\mathrm{M}}$ and $\boldsymbol{\varphi} \mathrm{NO}$ values for WT and autophagy mutants at $12 \mathrm{~h}$ and $24 \mathrm{~h}$. C. Relative apparent 1019 electron transport rate (ETR) plots for WT and autophagy mutants at $12 \mathrm{~h}$ and $24 \mathrm{~h}$. The grey regression represents $+/-95 \%$ confidence level intervals for prediction of the local regression. $\mathrm{H}$ and L, represent significantly higher values for WT with respect to autophagy mutants in terms of the height $(\mathrm{H})$ and length $(\mathrm{L})$ of the maximum ETR as explained in Figure S3. D. Ratio between photosynthetic activity and respiration for WT and autophagy mutants at $0 \mathrm{~h}$, 
$12 \mathrm{~h}$ and $24 \mathrm{~h}$. Asterisks indicate statistically significant differences $(\mathrm{P}<0.05)$ by Tukey's HSD test compared to WT. Data are represented as mean \pm S.D.

Figure 4. Differentially enriched plastid proteins in autophagy mutants during the dark-light transition. A. Venn diagrams for DEPPs at 0, 12 and $24 \mathrm{~h}$ for the individual autophagy mutants. B. Venn diagrams for DEPPs common to at least three atg lines at the indicated times. C. Enrichment of metabolic pathways and biological processes for the DEPPs of autophagy mutants that were consistent in at least two time points. The horizontal bars behind the text indicate the Enrichment score for each BP.

Figure 5. Heatmap and list of differentially enriched plastid proteins in autophagy mutants during the dark-light transition. The colours indicate the Z-score, which in some cases reached values much lower than -3 (e.g. -7) but were set from -3 to +3 to be more comprehensive. Grey colour indicates missing data. The hierarchical clustering generated two clear clusters which were separated in the figure as less and more abundant for visual purpose only.

Figure 6. Correlation between proteomic data and transcriptomic data for differentially enriched plastid proteins. A. Heatmap showing the $\log 2(\mathrm{FC})$ for the proteomic and transcriptomic data of each autophagy mutant relative to WT at 0,12 and $12 \mathrm{~h}$. B. Correlation graphs expressing transcriptomic vs proteomic data as normalized $\log 2(\mathrm{TPM})$ vs normalized $\log 2$ (LFQ) for the DEPP in WT and atg lines at 0, 12 and 24h. The numbers in the right bottom of each graph indicates the Pearson correlation coefficient and the colours indicate lowest (red) and greatest (blue) values. The asterisks in the top right corner indicate the pvalue as follows $*$, p-value $<0.05$ and $* *$, p-value $<0.01$.

Figure 7. A. Green Index for WT, autophagy and lipid mutants, and autophagy/lipid double mutants at $0 \mathrm{~h}, 6 \mathrm{~h}, 12 \mathrm{~h}$ and $24 \mathrm{~h}$ of light treatment. B. Ratio between photosynthetic activity and respiration for WT, autophagy and lipid mutants, and autophagy/lipid double mutants at $12 \mathrm{~h}$ and $24 \mathrm{~h}$. C. Analysis of $\mathrm{F}_{\mathrm{V}} / \mathrm{F}_{\mathrm{M}}$ values for WT, autophagy and lipid mutants, and autophagy/lipid double mutants at $6 \mathrm{~h}, 12 \mathrm{~h}$ and $24 \mathrm{~h}$. D. Relative apparent electron transport rate (ETR) plots for WT, autophagy and lipid mutants, and autophagy/lipid double mutants at $12 \mathrm{~h}$ and $24 \mathrm{~h}$. The grey regression represents $+/-99 \%$ confidence level intervals for prediction for the polynomial regression and $\mathrm{H}$, and $\mathrm{L}$, represent respectively significantly higher values for $W T$ respect to autophagy mutants in terms of the height $(\mathrm{H})$ and length $(\mathrm{L})$ of the maximum ETR as explained in Figure S3 and S5. Asterisks indicate statistically significant differences $(\mathrm{P}<0.05)$ by Tukey's HSD test compared to WT.

1063 Figure 8. High carbon source $(C)$ medium suppresses the delay in photomorphogenesis 1064 observed in autophagy mutants, whereas low $C$ medium worsens the atg phenotype. A. Green Index under different $C$ nutrient regimes. B. Analysis of $F_{V} / F_{M}$ under different $C$ nutrient regimes. C. Relative apparent electron transport rate (ETR) plots for WT and autophagy 
1068 illumination in mediums containing $0.1 \%, 0.5 \%$ and $2.0 \%$ sucrose. Asterisks indicate

1069 statistically significant differences $(\mathrm{P}<0.05)$ by Tukey's HSD test compared to WT. For 1070 ETR, the grey regression represents +/- 95\% confidence level intervals for prediction for the 1071 local regression and $\mathrm{H}$, and $\mathrm{L}$, represent respectively significantly higher values for $W T$ 1072 respect to autophagy mutants in terms of the height $(\mathrm{H})$ and length $(\mathrm{L})$ of the maximum ETR 1073 as explained in Figure S3 and S6.

1075 Figure 9. Mechanistic insights into the autophagy effect on photomorphogenesis and its 1076 relation with the current literature. The scheme describes the main mechanism discussed 1077 here by which autophagy could contribute to photomorphogenesis. The first mechanism is the 1078 contribution of autophagy to phosphatidylcholine recycling via organellophagy, which is 1079 used for thylakoid assembly during chloroplast development. We showed that tgdl lines 1080 presented a delayed greening (Fig. 7) supporting the mechanism enclosed in (A). The second 1081 mechanism involves the contribution of autophagy to fatty acids release, either through 1082 organellophagy or lipophagy. Both mechanisms converge in the production of sugars, either 1083 through photosynthesis or FA degradation, which in turn are known to support 1084 photomorphogenesis both energetically and as signalling molecules allowing TOR activity. Through the $s d p l$ lines, we showed that lipolysis is indeed relevant for photomorphogenesis (Fig. 7) supporting the mechanism enclosed in (B). Finally, we showed that sugar levels can 1088 Eastmond 2006; [3]: Li-Beisson et al. 2013; [4]: Michaud et al. 2019; [5]: LeBrant et al. 1089 2018; [6]: Xu et al. 2003; [7]: Xu et al. 2010; [8]: Li et al. 2018; [9]: Schnurr et al. 2002; 1090 [10]: Baena-Gonzalez et al. 2007; [11]: Ghillebert et al. 2011; [12]: Nukarinen et al. 2016; 1091 [13]: Chen et al. 2018; [14]: Cai et al. 2017; [15]: Pfeiffer et al. 2016. Cp: chloroplasts; ER: 1092 endoplasmic reticulum; DAG: diacylglycerol; DGDG: digalactosyldiacylglycerol; FA: fatty 1093 acids; LD: lipid droplets; MGDG: monogalactosyldiacylglycerol; Mt: mitochondria; PA: 1094 phosphatidate; PC: phosphatidylcholine; PE: phosphatidylethanolamine; PI: 1095 phosphatidylinositol; Pm: plasma membrane; Px: peroxisome; SnRK1: Snf1-related kinase 1; 1096 TAG: triacylglycerols; TOR: target of rapamycin. 


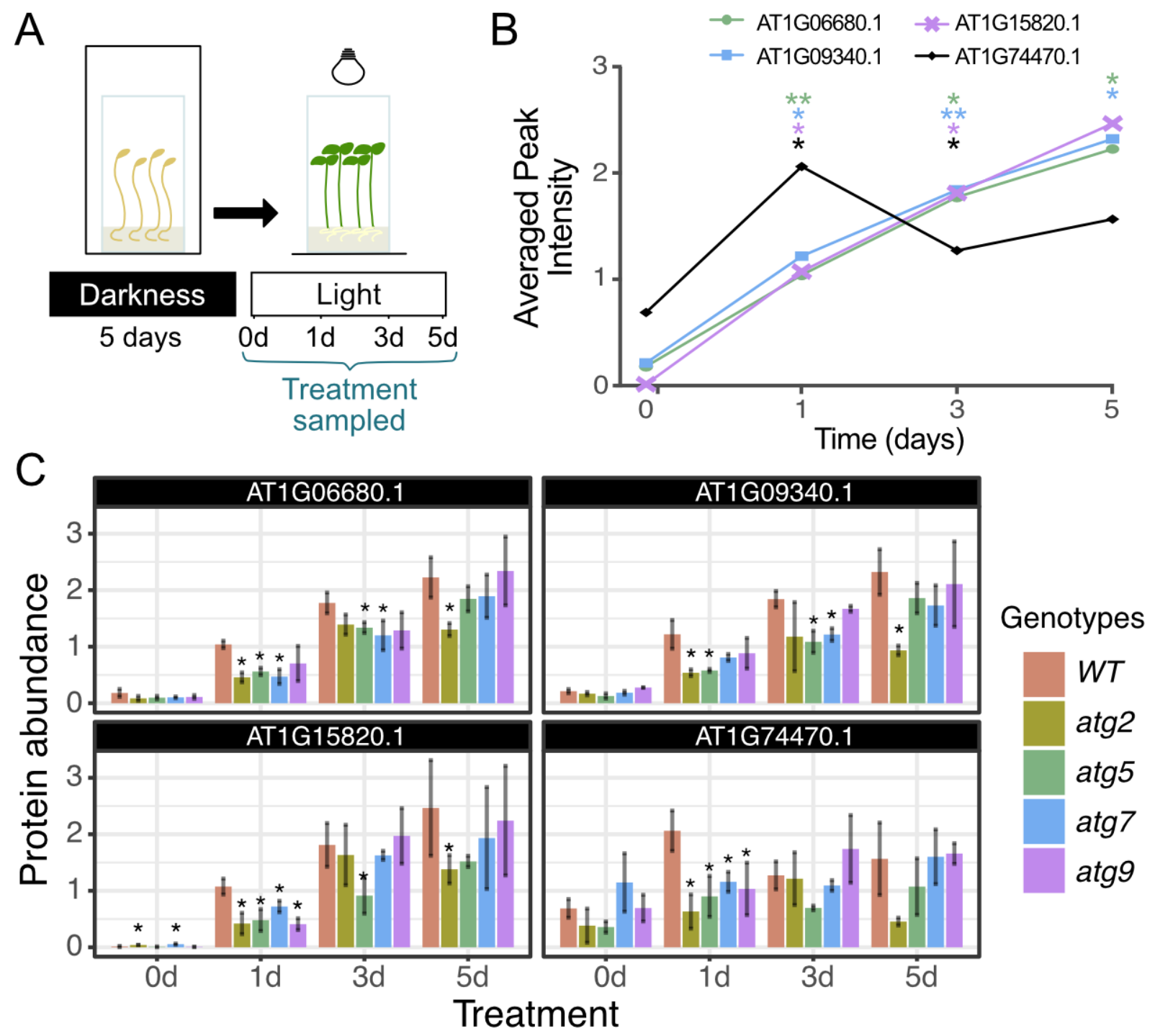

Figure 1. The dark to light transition for seedling greening is affected in atg lines and characterized by less abundant chloroplast markers. A. Experimental design showing the dark to light transition. B. Abundance of plastid MRM biomarkers, viz. AT1G06680.1 (PHOTOSYSTEM II SUBUNIT P-1), AT1G09340.1 (CHLOROPLAST RNA BINDING), AT1G15820.1 (LIGHT HARVESTING COMPLEX B6 OF PHOTOSYSTEM II), and AT1G74470.1 (GERANYLGERANYL DIPHOSPHATE REDUCTASE), during the dark to light transition in WT. The y-axis of the line graph represents averaged peak intensity of three biological samples $(n=3)$ of these markers over the time course. Statistical analyses were performed by one-way ANOVA, followed by Tukey's HSD test compared to the $0 \mathrm{~d}$ time $(* \mathrm{P}$ $<0.05$,). C. Abundance of plastid MRM biomarkers in WT Col-0 and autophagy mutants $\operatorname{atg} 2, \operatorname{atg} 5, \operatorname{atg} 7$ and $\operatorname{atg} 9$ during the dark to light transition $(0,1,3$ and 5 days). Means \pm SE for $n=4$ are shown. Asterisks denote statistically significant differences at the $\mathrm{P}<0.05$ level among treatment groups within a time-point. 
A

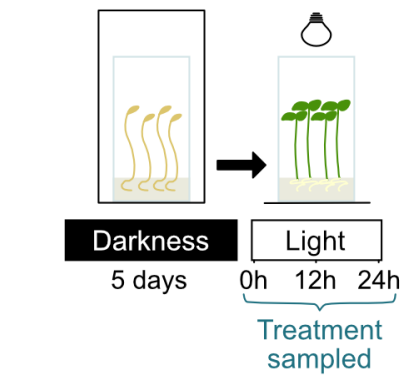

C

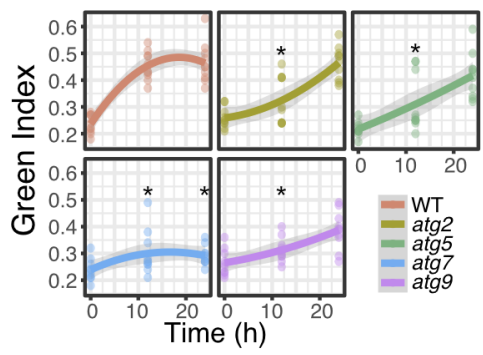

$\mathbf{F}$

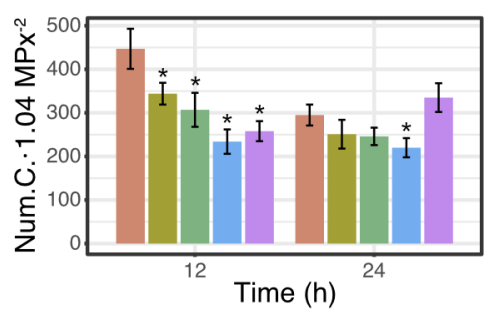

B

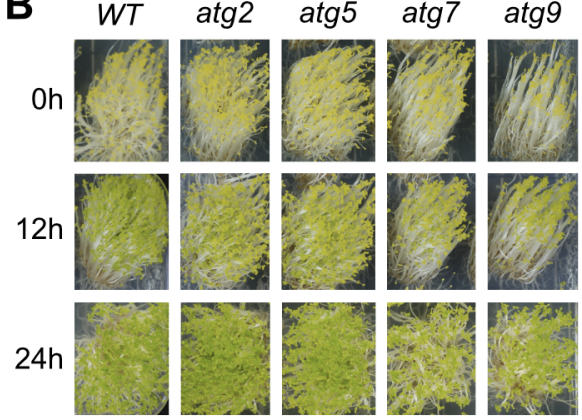

D

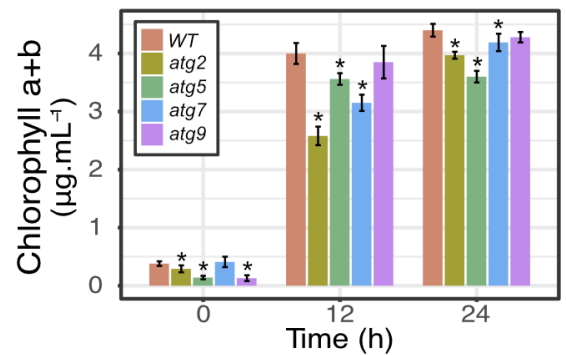

G

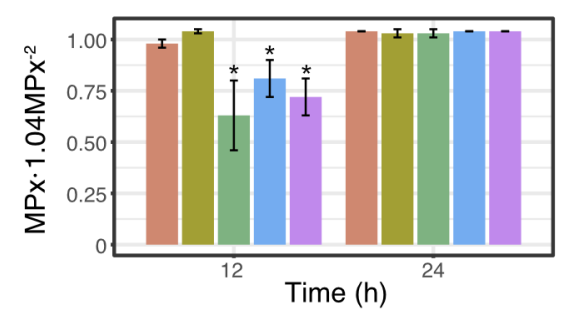

E
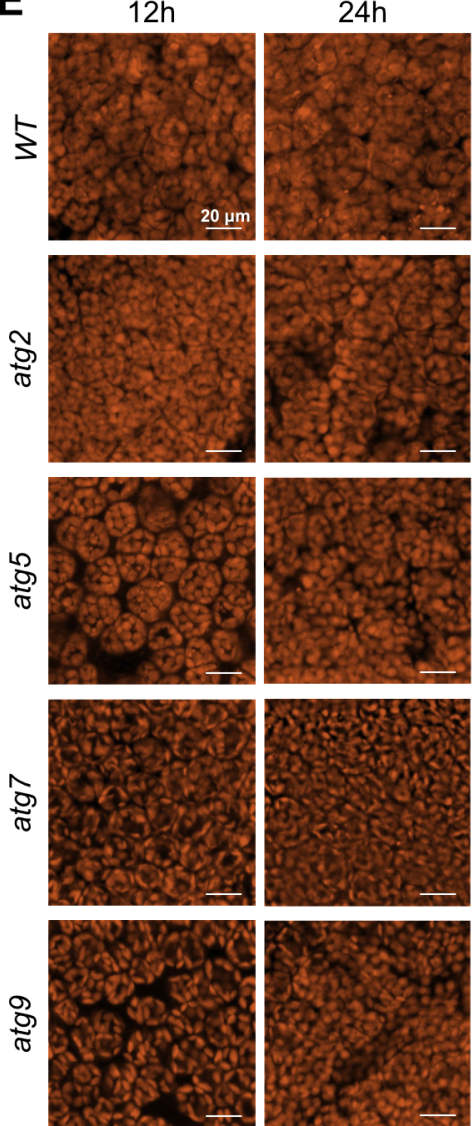

Figure 2. The dark to light transition in autophagy mutants is characterized by slower greening, lower chlorophyll content, and reduced number and size of chloroplast than the WT. A. Experimental design to study the dark to light transition for seedling greening. Seeds were germinated and grown on MS solid medium supplemented with $0.5 \%$ sucrose for 5 days in dark and then exposed to light for $0 \mathrm{~h}, 12 \mathrm{~h}$ and $24 \mathrm{~h}$. B. Phenotype of WT and autophagy mutants at $0 \mathrm{~h}, 12 \mathrm{~h}$ and $24 \mathrm{~h}$ of light treatment. C. Green Index for WT and autophagy mutants at $0 \mathrm{~h}, 12 \mathrm{~h}$ and $24 \mathrm{~h}$ of light treatment. The grey regression represents $+/-95 \%$ confidence level intervals for prediction for the 2-order polynomial regression. D. Chlorophyll content for WT and autophagy mutants at $0 \mathrm{~h}, 12 \mathrm{~h}$ and $24 \mathrm{~h}$ of light treatment. $(\mathrm{n}=4$ independent biological replicates with three technical replicates per group). E. Chlorophyll autofluorescence of WT and autophagy mutants at $12 \mathrm{~h}$ and $24 \mathrm{~h}$ of light treatment. Autofluorescence was detected by CLSM at $640 \mathrm{~nm}$ using a excitation of $488 \mathrm{~nm}$ (scale bars: $20 \mu \mathrm{m}$ ). F. Number of plastids per area, and G. plastid area for WT and autophagy mutants at $12 \mathrm{~h}$ and $24 \mathrm{~h}$ of light treatment. Resolution size $0.07 \mu \mathrm{m} / \mathrm{px}$, dimension of $1024 \mathrm{x} 1024 \mathrm{px}$ (equivalent to 1.04 $\left.\mathrm{MPx}^{2}\right)$ of autophagy mutants with control WT are shown ( $\mathrm{n}=5$ independent biological replicates per group). Asterisks indicate $\mathrm{P}$ values $\leq 0.05$ by two-way analysis of variance (ANOVA) and Tukey HSD multiple comparison test. Data are represented as mean \pm S.D. 
A
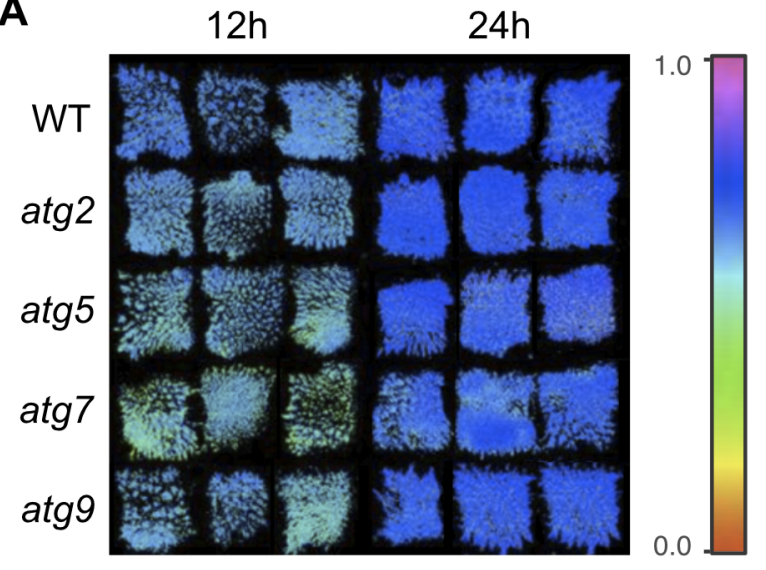

C

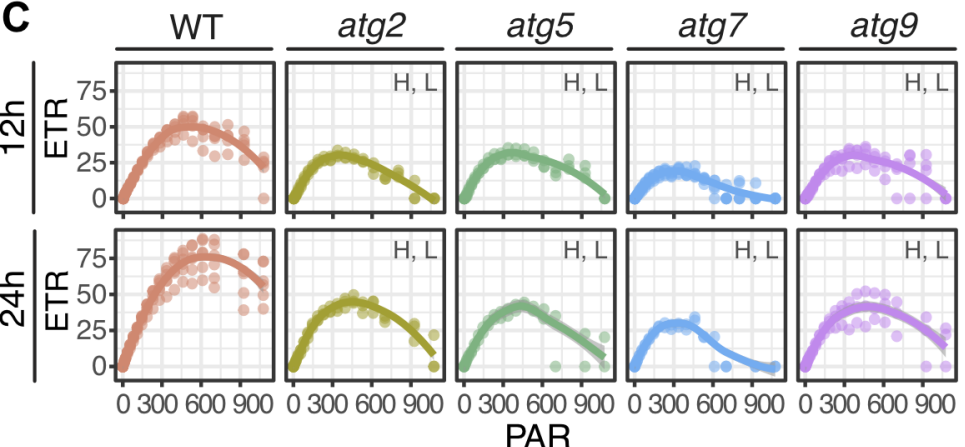

B
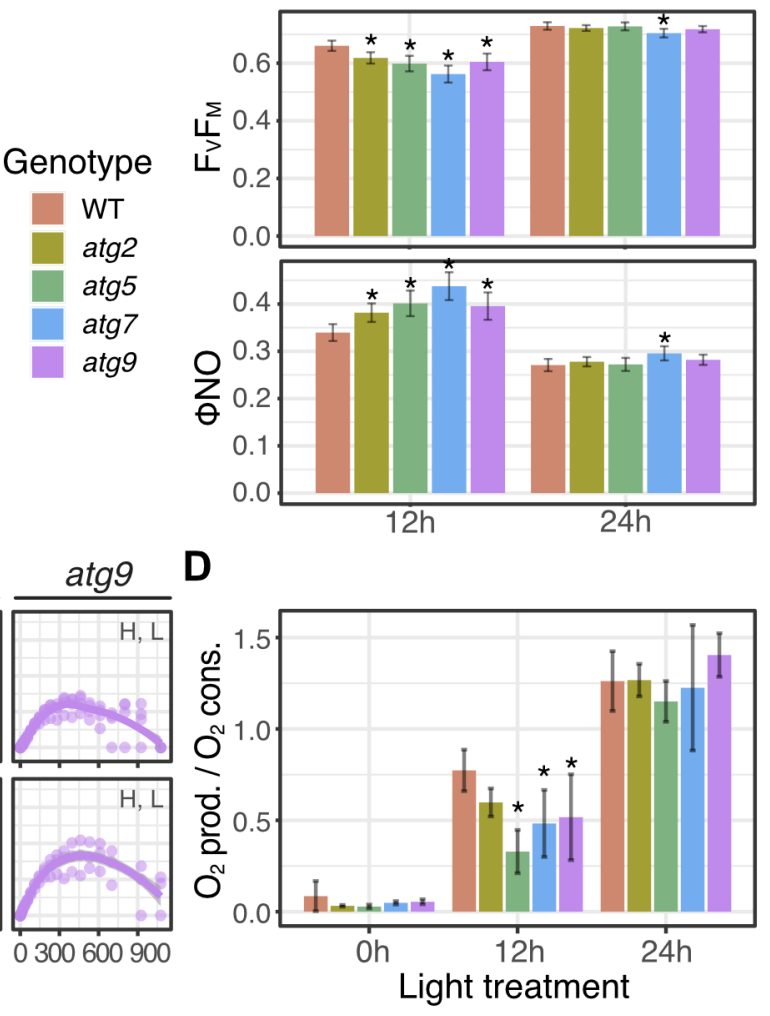

Figure 3. Photosynthetic performance of autophagy mutants during the dark to light transition. A. $\mathrm{F}_{\mathrm{V}} / \mathrm{F}_{\mathrm{M}}$ images for WT and autophagy mutants at $12 \mathrm{~h}$ and $24 \mathrm{~h}$. B. Analysis of $\mathrm{F}_{\mathrm{V}} / \mathrm{F}_{\mathrm{M}}$ and $\boldsymbol{\varphi}$ values for WT and autophagy mutants at $12 \mathrm{~h}$ and $24 \mathrm{~h}$. C. Relative apparent electron transport rate (ETR) plots for WT and autophagy mutants at $12 \mathrm{~h}$ and $24 \mathrm{~h}$. The grey regression represents $+/-95 \%$ confidence level intervals for prediction of the local regression. $\mathrm{H}$ and $\mathrm{L}$, represent significantly higher values for WT with respect to autophagy mutants in terms of the height $(\mathrm{H})$ and length (L) of the maximum ETR as explained in Figure S3. D. Ratio between photosynthetic activity and respiration for WT and autophagy mutants at $0 \mathrm{~h}, 12 \mathrm{~h}$ and $24 \mathrm{~h}$. Asterisks indicate statistically significant differences $(\mathrm{P}<0.05)$ by Tukey's HSD test compared to WT. Data are represented as mean \pm S.D. 
A
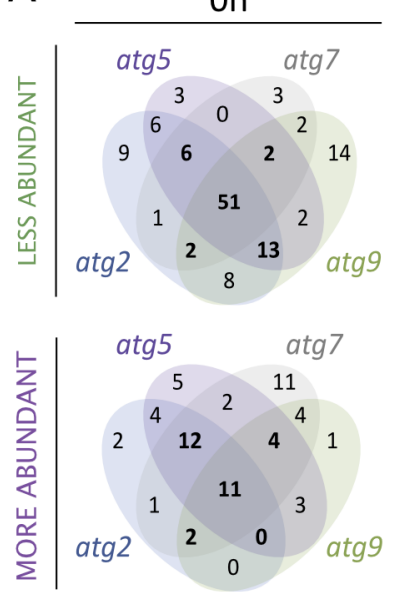

$12 \mathrm{~h}$
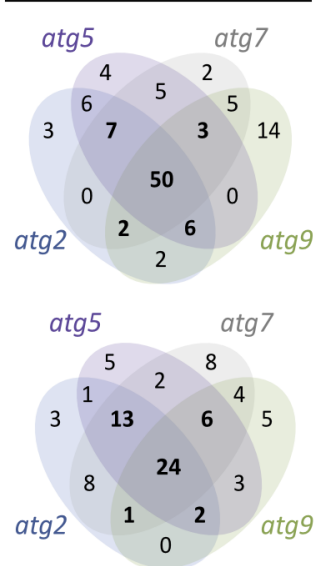

$24 \mathrm{~h}$
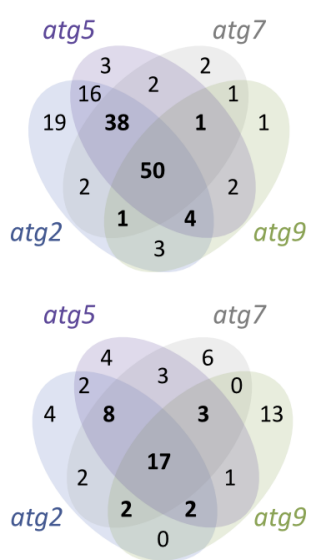

B consistent in 3 or 4 atgs
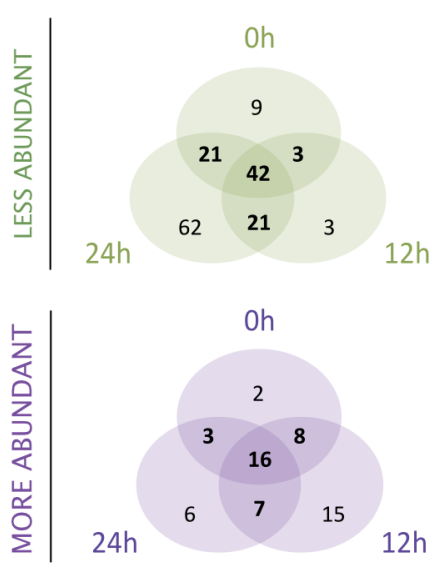

C

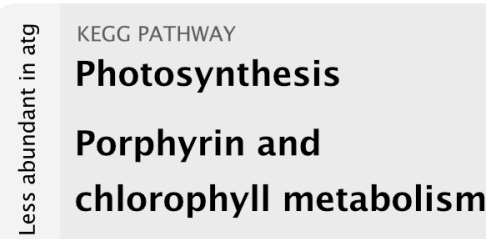

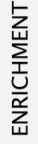

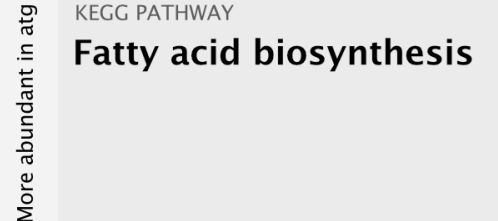

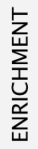

\begin{tabular}{lll}
\hline GO BIOLOGICAL PROCESS & \multicolumn{3}{l}{ Intersections } \\
\hline Chloroplast organization & 6 & $7.1 \mathrm{E}-05$ \\
\hline Tetrapyrrole metabolic process & 7 & $2.9 \mathrm{E}-05$ \\
\hline Plastid organization & 7 & $2.9 \mathrm{E}-05$ \\
\hline Porphyrin-containing compound metabolic process & 7 & $2.9 \mathrm{E}-05$ \\
\hline Chlorophyll metabolic process & 7 & $2.9 \mathrm{E}-05$ \\
\hline Porphyrin-containing compound biosynthetic process & 6 & $1.2 \mathrm{E}-04$ \\
\hline Tetrapyrrole biosynthetic process & 6 & $1.2 \mathrm{E}-04$ \\
\hline Chlorophyll biosynthetic process & 6 & $1.2 \mathrm{E}-04$ \\
\hline Photosynthesis & 17 & $6.0 \mathrm{E}-11$ \\
\hline Pigment metabolic process & 7 & $1.7 \mathrm{E}-04$ \\
\hline Defense response to bacterium & 16 & $3.3 \mathrm{E}-07$ \\
\hline Response to bacterium & 17 & $4.5 \mathrm{E}-07$ \\
\hline Heterocycle biosynthetic process & 15 & $8.1 \mathrm{E}-06$ \\
\hline Cellular nitrogen compound biosynthetic process & 22 & $1.1 \mathrm{E}-07$ \\
\hline & & \\
GO BIOLOGICAL PROCESS & $1 \mathrm{ntersections}$ \\
\hline Fatty acid biosynthetic process & 7 & $9.5 \mathrm{E}-07$ \\
\hline Lipid biosynthetic process & 7 & $9.4 \mathrm{E}-06$ \\
\hline Fatty acid metabolic process & 7 & $2.8 \mathrm{E}-05$ \\
\hline Monocarboxylic acid metabolic process & 11 & $2.1 \mathrm{E}-07$ \\
\hline Glucose catabolic process & 7 & $6.8 \mathrm{E}-05$ \\
\hline Hexose catabolic process & 7 & $8.0 \mathrm{E}-05$ \\
\hline Glucose metabolic process & 8 & $2.7 \mathrm{E}-05$ \\
\hline Monosaccharide catabolic process & 7 & $9.4 \mathrm{E}-05$ \\
\hline Alcohol catabolic process & 7 & $1.1 \mathrm{E}-04$ \\
Cellular lipid metabolic process & 7 & $1.9 \mathrm{E}-04$ \\
\hline Hexose metabolic process & 8 & $7.5 \mathrm{E}-05$ \\
\hline Organic acid biosynthetic process & 10 & $1.1 \mathrm{E}-05$ \\
\hline Carboxylic acid biosynthetic process & 10 & $1.1 \mathrm{E}-05$ \\
\hline Monosaccharide metabolic process & 9 & $7.5 \mathrm{E}-05$ \\
\hline Small molecule biosynthetic process & 11 & $1.0 \mathrm{E}-05$ \\
\hline Carboxylic acid metabolic process & 14 & $3.2 \mathrm{E}-05$ \\
Oxoacid metabolic process & 14 & $3.2 \mathrm{E}-05$ \\
\hline Cellular ketone metabolic process & 14 & $3.4 \mathrm{E}-05$ \\
Organic acid metabolic process & $3.4 \mathrm{E}-05$ \\
\hline
\end{tabular}

Figure 4. Differentially enriched plastid proteins in autophagy mutants during the dark-light transition. A. Venn diagrams for DEPPs at 0,12 and $24 \mathrm{~h}$ for the individual autophagy mutants. B. Venn diagrams for DEPPs common to at least three atg lines at the indicated times. C. Enrichment of metabolic pathways and biological processes for the DEPPs of autophagy mutants that were consistent in at least two time points. The horizontal bars behind the text indicate the Enrichment score for each BP. 


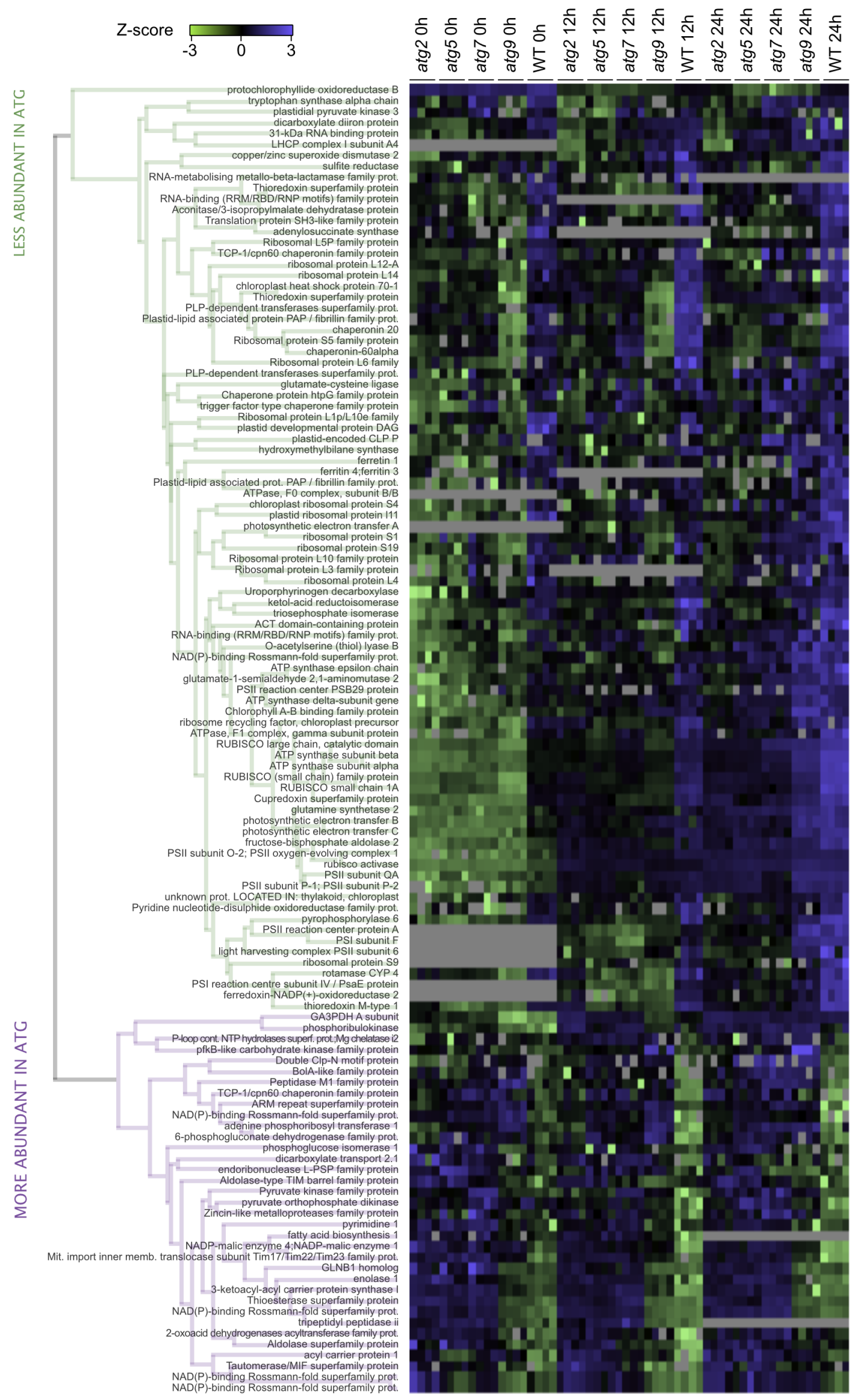

Figure 5. Heatmap and list of differentially enriched plastid proteins in autophagy mutants during the dark-light transition. The colours indicate the Z-score, which in some cases reached values much lower than -3 (e.g. -7) but were set from -3 to +3 to be more comprehensive. Grey colour indicates missing data. The hierarchical clustering generated two clear clusters which were separated in the figure as less and more abundant for visual purpose only. 
A

\begin{tabular}{|l|lll}
\hline \multicolumn{2}{|c|}{ Proteins } & \multicolumn{2}{c|}{ Transcripts } \\
\hline Oh $\quad 12 \mathrm{~h} \quad 24 \mathrm{~h}$ & Oh $\quad 12 \mathrm{~h} \quad 24 \mathrm{~h}$ \\
\hline
\end{tabular}

AT1603680

AT1605190

AT1G11430

AT1G32990

AT1644575;

AT1667280

AT2G04030;

AT2628000

AT2G28190

AT2 233800
AT2G37220

AT2G37660

AT2G39730;

AT2G43750;

AT3G11630

AT3G23400
AT3G48730

AT3G54640

AT $3656940 ;$
AT 3658610

AT3G63190

AT3663490

AT4G01310

AT4 4603520

AT4609650

AT4G21280

AT4G23100

AT4G24280

AT4G 24770

AT4G33680

AT4G38970;

AT5G04590

ATS608280

ATS 609650

AT5G20720

AT5G30510

AT5G35630;

AT5G54600

AT5G58250

ATCG00120

ATCG00470

ATCG00480

ATCG00720

ATCG00820

AT1G24360

AT1G27450;

AT1G32060

AT1G54870

AT1664190;

AT1664190

AT1674030

AT2G01140

AT2605990;

AT2619900;

AT3G05020

AT3G17810

AT3G20390

AT3G22960

AT3G26650

AT4G01900

AT4G15530;
AT4G16160;

AT4G16160;

AT4G18480;

AT5G01650

AT5G13420
AT5G17560

AT5G46290;

AT5G53480

AT5G56500;

AT5G64290

AT5G65620

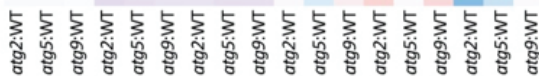

$\log 2(\mathrm{FC})$

Less abundant in atg

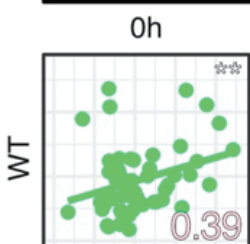

$12 \mathrm{~h}$
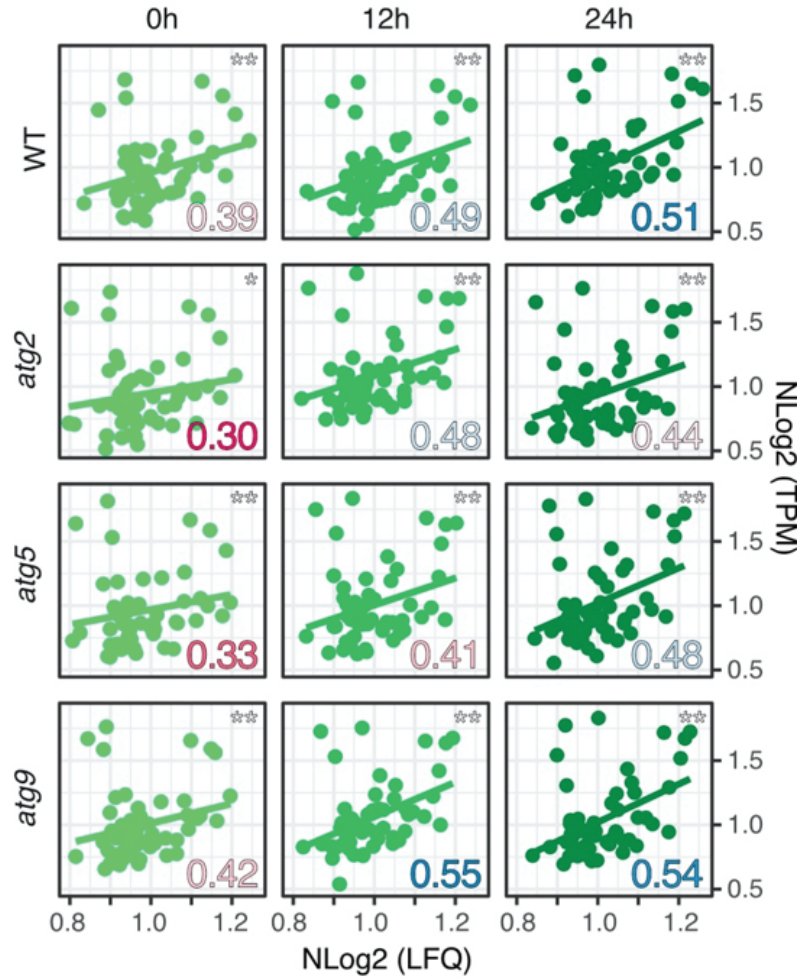

More abundant in atg

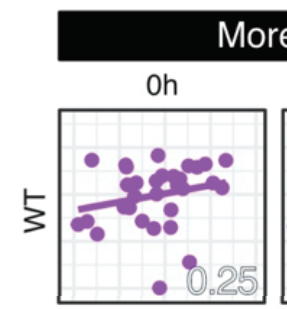

$12 \mathrm{~h}$
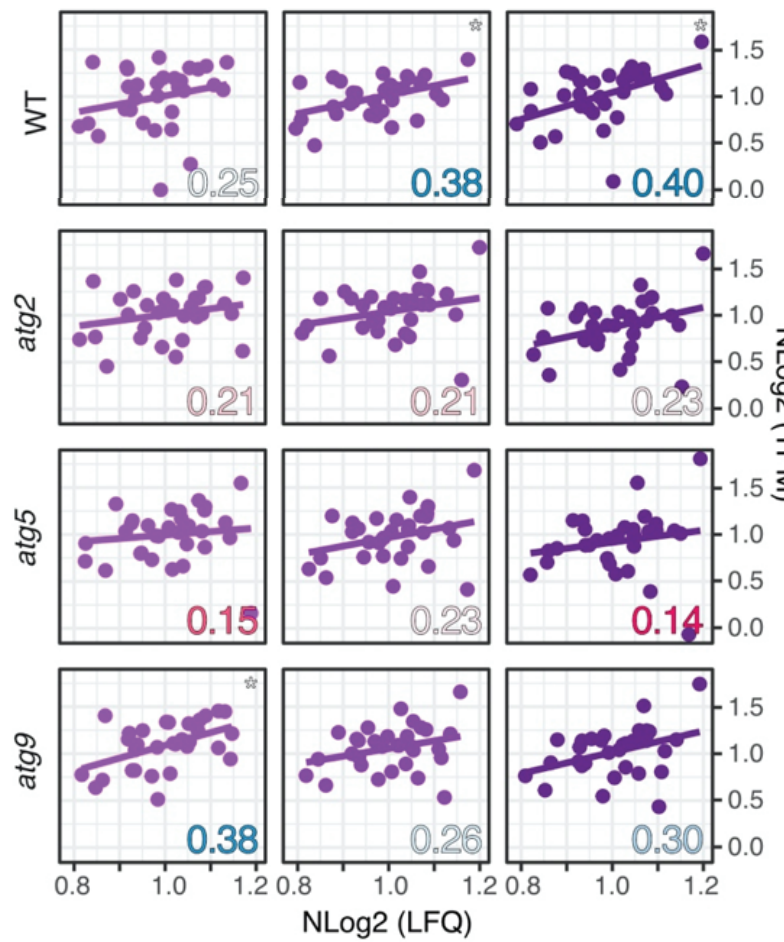

Figure 6. Correlation between proteomic data and transcriptomic data for differentially enriched plastid proteins. A. Heatmap showing the $\log 2(\mathrm{FC})$ for the proteomic and transcriptomic data of each autophagy mutant relative to WT at 0,12 and $12 \mathrm{~h}$. B. Correlation graphs expressing transcriptomic vs proteomic data as normalized Log2(TPM) vs normalized Log2(LFQ) for the DEPP in WT and atg lines at 0,12 and 24h. The numbers in the right bottom of each graph indicates the Pearson correlation coefficient and the colours indicate lowest (red) and greatest (blue) values. The asterisks in the top right corner indicate the p-value as follows $*$, p-value $<0.05$ and $* *$, p-value $<0.01$. 


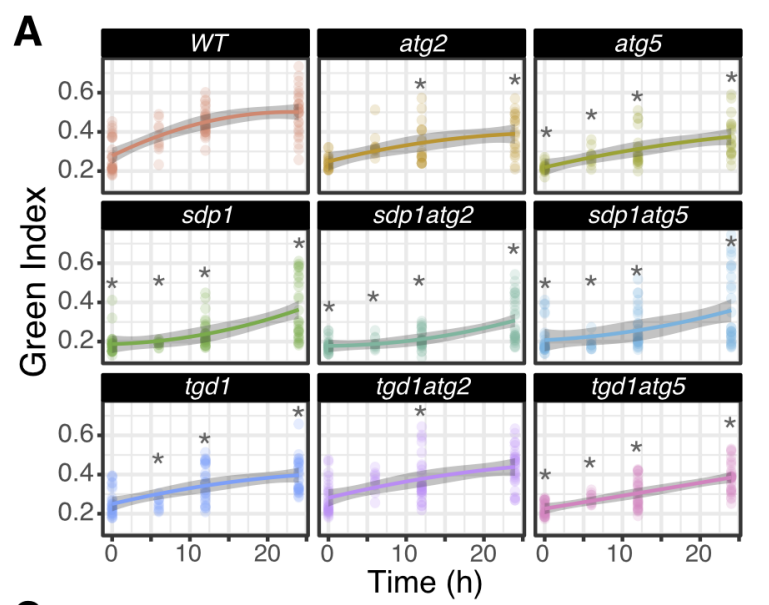

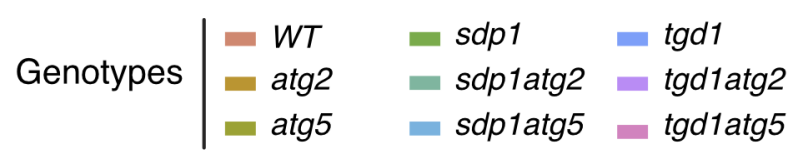

B
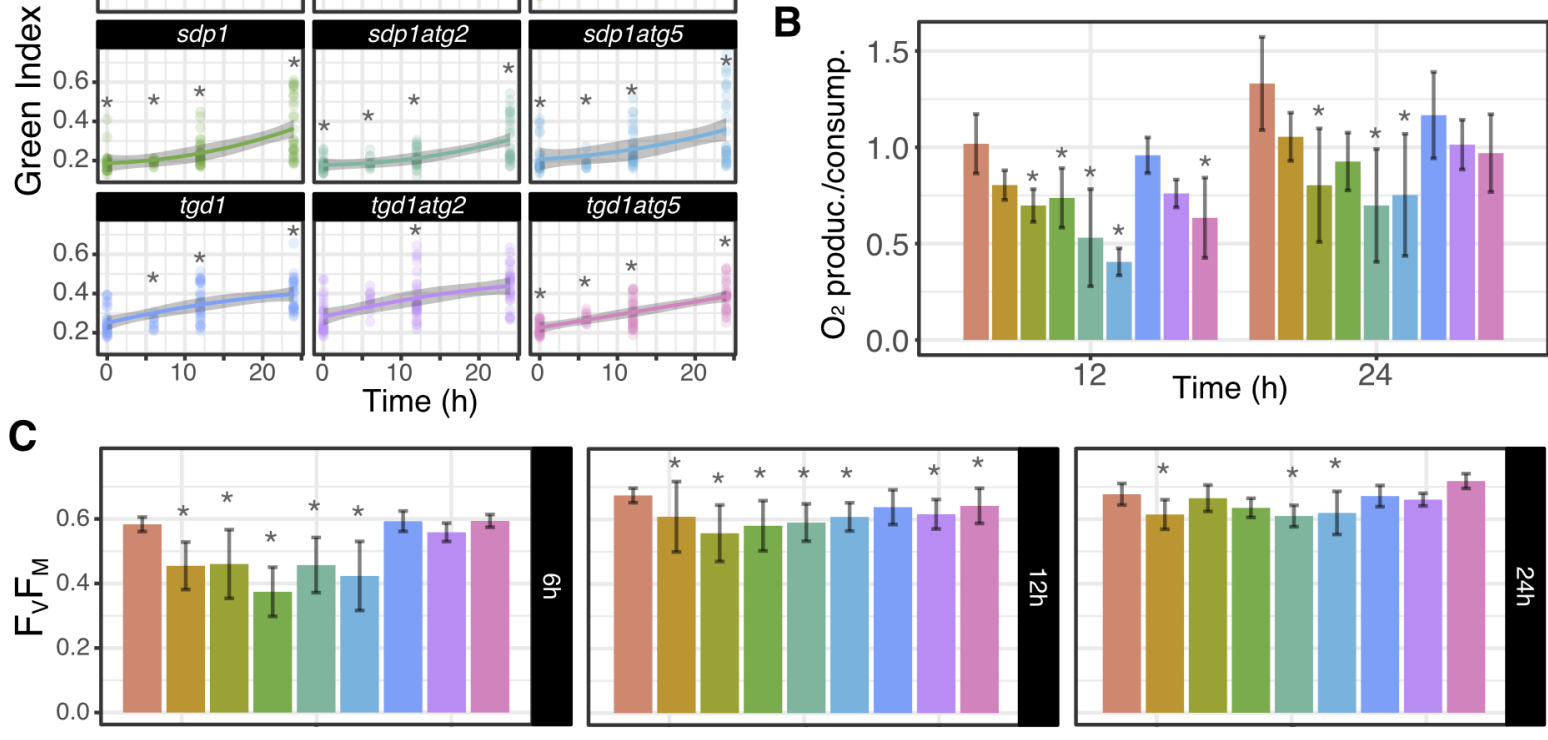

D
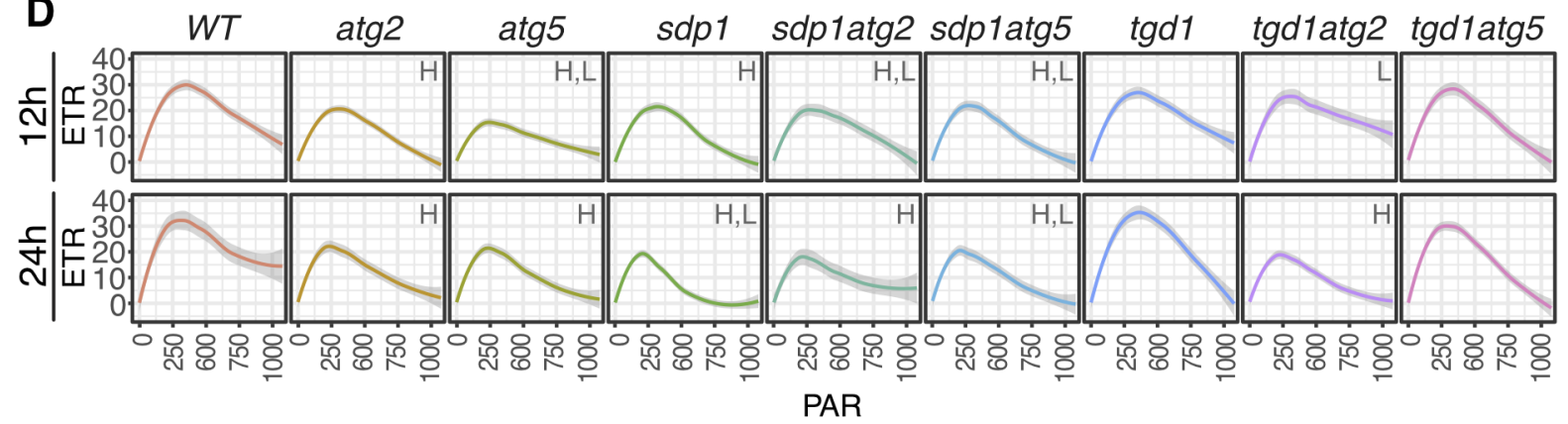

Figure 7. A. Green Index for WT, autophagy and lipid mutants, and autophagy/lipid double mutants at $0 \mathrm{~h}, 6 \mathrm{~h}, 12 \mathrm{~h}$ and $24 \mathrm{~h}$ of light treatment. B. Ratio between photosynthetic activity and respiration for WT, autophagy and lipid mutants, and autophagy/lipid double mutants at $12 \mathrm{~h}$ and $24 \mathrm{~h}$. C. Analysis of $\mathrm{F}_{\mathrm{V}} / \mathrm{F}_{\mathrm{M}}$ values for WT, autophagy and lipid mutants, and autophagy/lipid double mutants at $6 \mathrm{~h}, 12 \mathrm{~h}$ and $24 \mathrm{~h}$. D. Relative apparent electron transport rate (ETR) plots for WT, autophagy and lipid mutants, and autophagy/lipid double mutants at $12 \mathrm{~h}$ and $24 \mathrm{~h}$. The grey regression represents $+/-99 \%$ confidence level intervals for prediction for the polynomial regression and $\mathrm{H}$, and L, represent respectively significantly higher values for $W T$ respect to autophagy mutants in terms of the height $(\mathrm{H})$ and length $(\mathrm{L})$ of the maximum ETR as explained in Figure S3 and S5. Asterisks indicate statistically significant differences $(\mathrm{P}<0.05)$ by Tukey's HSD test compared to WT. 

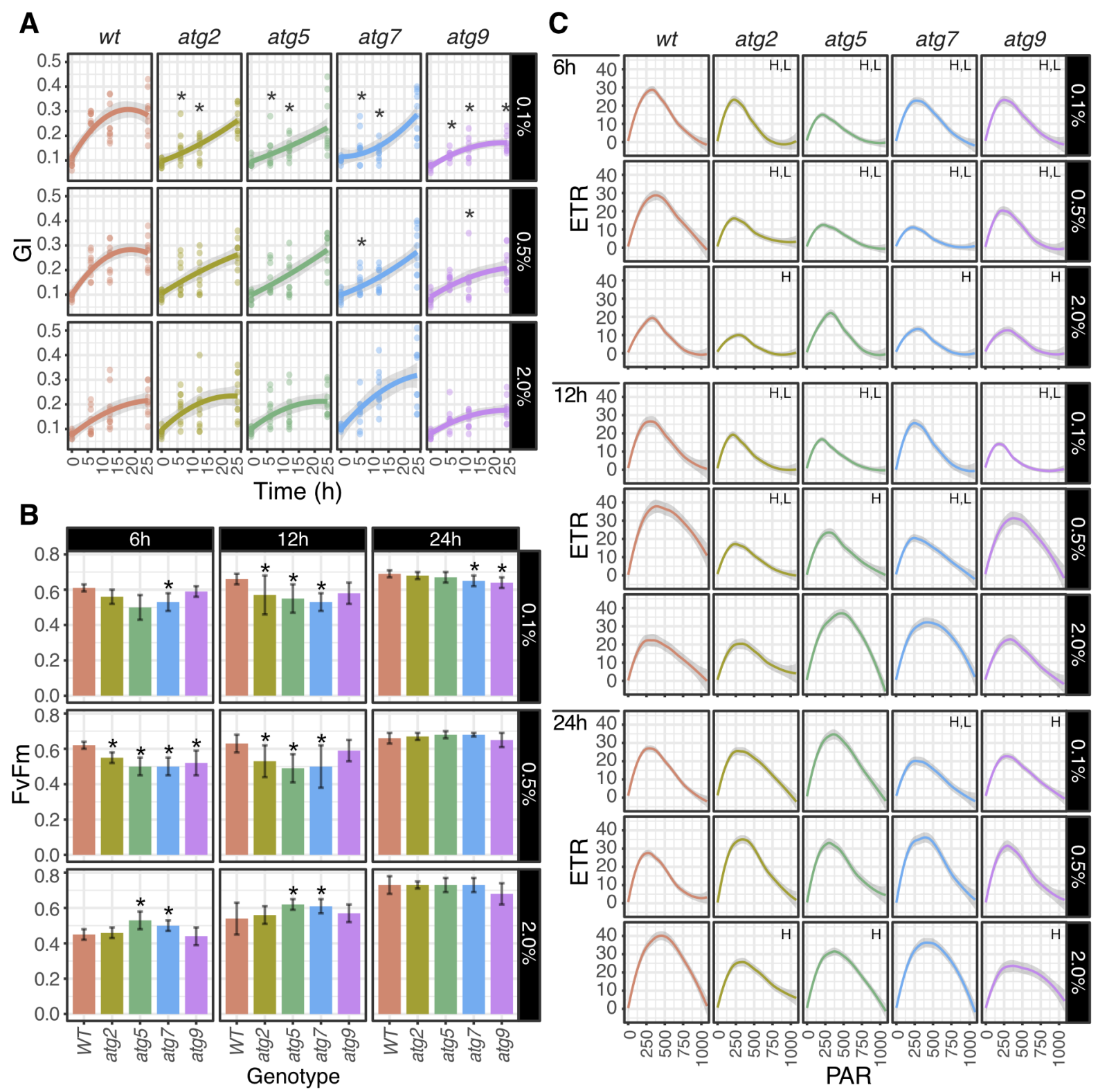

Figure 8. High carbon source (C) medium suppresses the delay in photomorphogenesis observed in autophagy mutants, whereas low $C$ medium worsens the atg phenotype. A. Green Index under different $\mathrm{C}$ nutrient regimes. B. Analysis of $\mathrm{F}_{\mathrm{V}} / \mathrm{F}_{\mathrm{M}}$ under different $\mathrm{C}$ nutrient regimes. C. Relative apparent electron transport rate (ETR) plots for WT and autophagy mutants. In all the cases the genotypes were evaluated at $0 \mathrm{~h}, 6 \mathrm{~h}, 12 \mathrm{~h}$ and $24 \mathrm{~h}$ post illumination in mediums containing $0.1 \%, 0.5 \%$ and $2.0 \%$ sucrose. Asterisks indicate statistically significant differences $(\mathrm{P}<0.05)$ by Tukey's HSD test compared to WT. For ETR, the grey regression represents $+/-95 \%$ confidence level intervals for prediction for the local regression and $\mathrm{H}$, and $\mathrm{L}$, represent respectively significantly higher values for $W T$ respect to autophagy mutants in terms of the height $(\mathrm{H})$ and length $(\mathrm{L})$ of the maximum ETR as explained in Figure S3 and S6. 


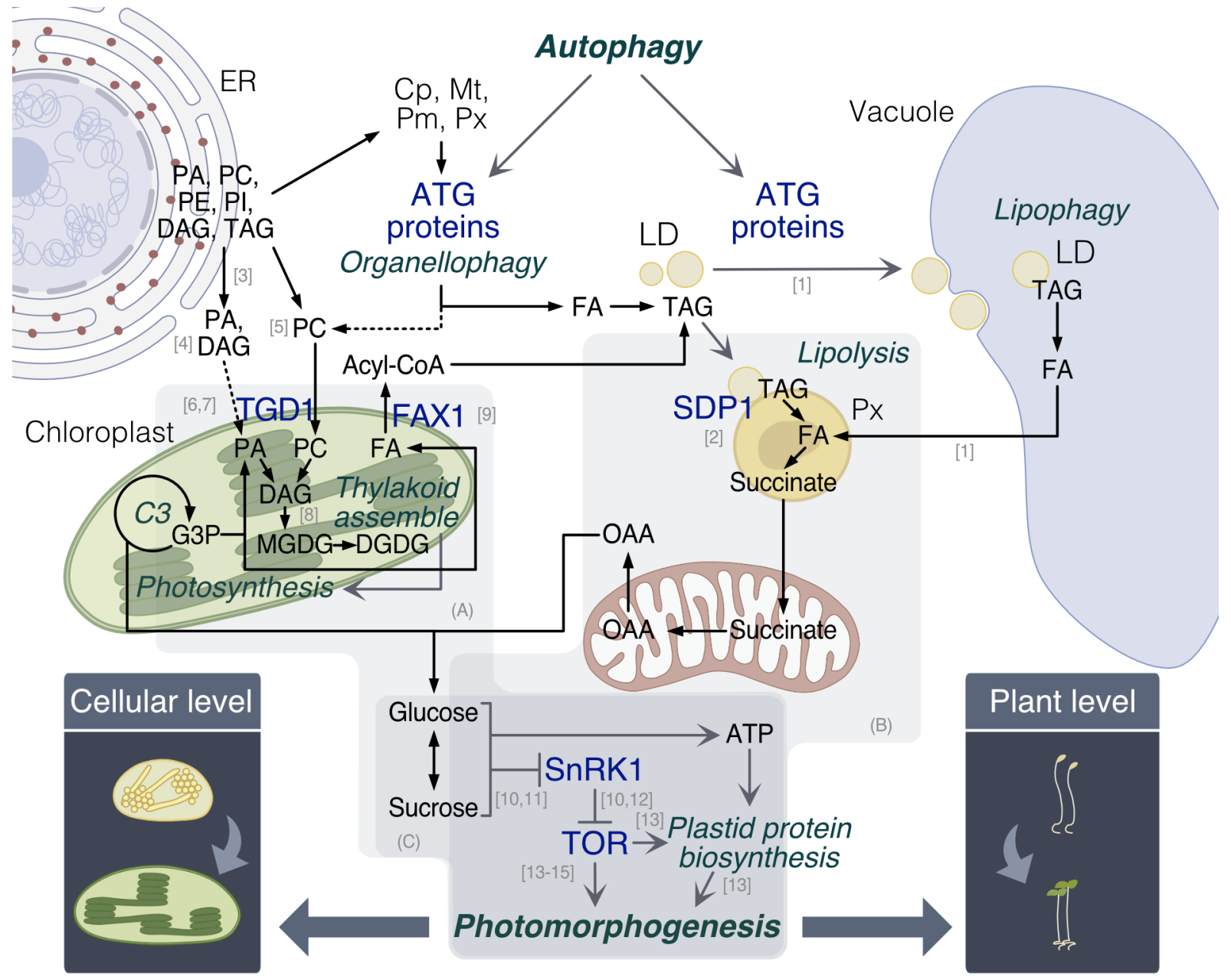

Figure 9. Mechanistic insights into the autophagy effect on photomorphogenesis and its relation with the current literature. The scheme describes the main mechanism discussed here by which autophagy could contribute to photomorphogenesis. The first mechanism is the contribution of autophagy to phosphatidylcholine recycling via organellophagy, which is used for thylakoid assembly during chloroplast development. We showed that tgdl lines presented a delayed greening (Fig. 7) supporting the mechanism enclosed in (A). The second mechanism involves the contribution of autophagy to fatty acids release, either through organellophagy or lipophagy. Both mechanisms converge in the production of sugars, either through photosynthesis or FA degradation, which in turn are known to support photomorphogenesis both energetically and as signalling molecules allowing TOR activity. Through the $s d p 1$ lines, we showed that lipolysis is indeed relevant for photomorphogenesis (Fig. 7) supporting the mechanism enclosed in (B). Finally, we showed that sugar levels can affect the degree of photomorphogenesis (Fig. 8) as shown in (C). [1]: Fan et al. 2019; [2]: Eastmond 2006; [3]: Li-Beisson et al. 2013; [4]: Michaud et al. 2019; [5]: LeBrant et al. 2018; [6]: Xu et al. 2003; [7]: Xu et al. 2010; [8]: Li et al. 2018; [9]: Schnurr et al. 2002; [10]: Baena-Gonzalez et al. 2007; [11]: Ghillebert et al. 2011; [12]: Nukarinen et al. 2016; [13]: Chen et al. 2018; [14]: Cai et al. 2017; [15]: Pfeiffer et al. 2016. Cp: chloroplasts; ER: endoplasmic reticulum; DAG: diacylglycerol; DGDG: digalactosyldiacylglycerol; FA: fatty acids; LD: lipid droplets; MGDG: monogalactosyldiacylglycerol; Mt: mitochondria; PA: phosphatidate; PC: phosphatidylcholine; PE: phosphatidylethanolamine; PI: phosphatidylinositol; Pm: plasma membrane; Px: peroxisome; SnRK1: Snf1-related kinase 1; TAG: triacylglycerols; TOR: target of rapamycin. 


\section{Parsed Citations}

Armarego-Marriott, Kowalewska, Burgos, Fischer, Thiele, Erban, Strand, Kahlau, Hertle, Kopka, et al (2019) Highly resolved systems biology to dissect the etioplast-to-chloroplast transition in tobacco leaves. Plant Physiol 180:654-681

Google Scholar: Author Only Title Only Author and Title

Avin-Wittenberg (2018) Autophagy and its Role in Plant Abiotic Stress Management. Plant Cell Environ. https://doi.org/10.1111/pce.13404

Google Scholar: Author Only Title Only Author and Title

Avin-Wittenberg, Bajdzienko, Wittenberg, Aseekh, Tohge, Bock, Giavalisco, Fernie (2015) Global analysis of the role of autophagy in cellular metabolism and energy homeostasis in Arabidopsis seedlings under carbon starvation. Plant Cell 27:306-322

Google Scholar: Author Only Title Only Author and Title

Awai, Xu, Tamot, Benning (2006) A phosphatidic acid-binding protein of the chloroplast inner envelope membrane involved in lipid trafficking. Proc Natl Acad Sci U S A 103:10817-10822

Google Scholar: Author Only Title Only Author and Title

Baena-González, Rolland, Thevelein, Sheen (2007) Acentral integrator of transcription networks in plant stress and energy signalling. Nature 448:938-942

Google Scholar: Author Only Title Only Author and Title

Barbagallo, Oxborough, Pallett, Baker (2003) Rapid, noninvasive screening for perturbations of metabolism and plant growth using chlorophyll fluorescence imaging. Plant Physiol 132:483-493

Google Scholar: Author Only Title Only Author and Title

Bassham (2007) Plant autophagy - more than a starvation response. Curr Opin Plant Biol 10:587-593

Google Scholar: Author Only Title Only Author and Title

Bray, Pimentel, Melsted, Pachter (2016) Near-optimal probabilistic RNAseq quantification. Nat Biotechnol 34:525-527

Google Scholar: Author Only Title Only Author and Title

Broda, Millar, Van Aken (2018) Mitophagy: AMechanism for Plant Growth and Survival. Trends Plant Sci 23:434-450

Google Scholar: Author Only Title Only Author and Title

Cai, Li, Liu, Wang, Zhou, Xu, Xiong (2017) COP1 integrates light signals to ROP2 for cell cycle activation. Plant Signal Behav 12: Google Scholar: Author Only Title Only Author and Title

Calixto, Guo, James, Tzioutziou, Entizne, Panter, Knight, Nimmo, Zhang, Brown (2018) Rapid and dynamic alternative splicing impacts the arabidopsis cold response transcriptome[CC-BY]. Plant Cell 30:1424-1444

Google Scholar: Author Only Title Only Author and Title

Cao, Ni, Zhang, Shi, Xu, Yan, Zhang (2017) Atterations in the proteome of wheat primary roots after wortmannin application during seed germination. Acta Physiol Plant 39:223

Google Scholar: Author Only Title Only Author and Title

Chen, Liao, Qi, Xie, Huang, Tan, Zhai, Yuan, Zhou, Yu, et al (2015) Autophagy contributes to regulation of the hypoxia response during submergence in Arabidopsis thaliana. Autophagy 11:2233-2246

Google Scholar: Author Only Title Only Author and Title

Chen, Liu, Xiong, Sheen, Wu (2018) TOR and RPS6 transmit light signals to enhance protein translation in deetiolating Arabidopsis seedlings. Proc Natl Acad Sci U S A 115:12823-12828

Google Scholar: Author Only Title Only Author and Title

Cheng, Teo, Krueger, Rock, Koh, Choi, Vogel (2016) Differential dynamics of the mammalian mRNAand protein expression response to misfolding stress. Mol Syst Biol 12:855

Google Scholar: Author Only Title Only Author and Title

Chung, Suttangkakul, Vierstra (2009) The ATG autophagic conjugation system in maize: ATG transcripts and abundance of the ATG8lipid adduct are regulated by development and nutrient availability. Plant Physiol 149:220-234

Google Scholar: Author Only Title Only Author and Title

Cox, Mann (2008) MaxQuant enables high peptide identification rates, individualized p.p.b.-range mass accuracies and proteome-wide protein quantification. Nat Biotechnol 26:1367-1372

Google Scholar: Author Only Title Only Author and Title

Crisp, Ganguly, Smith, Murray, Estavillo, Searle, Ford, Bogdanović, Lister, Borevitz, et al (2017) Rapid recovery gene downregulation during excess-light stress and recovery in arabidopsis. Plant Cell 29:1836-1863

Google Scholar: Author Only Title Only Author and Title

Cui, Hayashi, Otomo, Mano, Oikawa, Hayashi, Nishimura (2016) Sucrose production mediated by lipid metabolism suppresses the physical interaction of peroxisomes and oil bodies during germination of arabidopsis thaliana. J Biol Chem 291:19734-19745

Google Scholar: Author Only Title Only Author and Title 
Di Berardino, Marmagne, Berger, Yoshimoto, Cueff, Chardon, Masclaux-Daubresse, Reisdorf-Cren (2018) Autophagy controls resource allocation and protein storage accumulation in Arabidopsis seeds. J Exp Bot 69:1403-1414

Google Scholar: Author Only Title Only Author and Title

Eastmond (2006) Sugar-dependent1 encodes a patatin domain triacylglycerol lipase that initiates storage oil breakdown in germinating Arabidopsis seeds. Plant Cell 18:665-675

Google Scholar: Author Only Title Only Author and Title

Fan, Yu, Xu (2017) A central role for triacylglycerol in membrane lipid breakdown, fatty acid $\beta$-oxidation, and plant survival under extended darkness. Plant Physiol 174:1517-1530

Google Scholar: Author Only Title Only Author and Title

Fan, Yu, Xu (2019) Dual role for autophagy in lipid metabolismin arabidopsis. Plant Cell 31:1598-1613

Google Scholar: Author Only Title Only Author and Title

Ghillebert, Swinnen, Wen, Vandesteene, Ramon, Norga, Rolland, Winderickx (2011) The AMPK/SNF1/SnRK1 fuel gauge and energy regulator: Structure, function and regulation. FEBS J 278:3978-3990

Google Scholar: Author Only Title Only Author and Title

Guiboileau, Yoshimoto, Soulay, Bataillé, Avice, Masclaux-Daubresse (2012) Autophagy machinery controls nitrogen remobilization at the whole-plant level under both limiting and ample nitrate conditions in Arabidopsis. New Phytol 194:732-740

Google Scholar: Author Only Title Only Author and Title

Guo, Tzioutziou, Stephen, Milne, Calixto, Waugh, Brown, Zhang (2020) 3D RNA-seq: a powerful and flexible tool for rapid and accurate differential expression and alternative splicing analysis of RNA-seq data for biologists. RNABiol.

https://doi.org/10.1080/15476286.2020.1858253

Google Scholar: Author Only Title Only Author and Title

Havé, Luo, Tellier, Balliau, Cueff, Chardon, Zvy, Rajjou, Cacas, Masclaux-Daubresse (2019) Proteomic and lipidomic analyses of the Arabidopsis atg5 autophagy mutant reveal major changes in endoplasmic reticulum and peroxisome metabolisms and in lipid composition. New Phytol 223:1461-1477

Google Scholar: Author Only Title Only Author and Title

Heredia-Martínez, Andrés-Garrido, Martínez-Force, Pérez-Pérez, Crespo (2018) Chloroplast damage induced by the inhibition of fatty acid synthesis triggers autophagy in chlamydomonas. Plant Physiol 178:1112-1129

Google Scholar: Author Only Title Only Author and Title

Hooper, Stevens, Saukkonen, Castleden, Singh, Mann, Fabre, Ito, Deery, Lilley, et al (2017) Multiple marker abundance profiling: combining selected reaction monitoring and data-dependent acquisition for rapid estimation of organelle abundance in subcellular samples. Plant J 92:1202-1217

Google Scholar: Author Only Title Only Author and Title

Ishida, Izumi, Wada, Makino (2014) Roles of autophagy in chloroplast recycling. Biochim Biophys Acta - Bioenerg 1837:512-521

Google Scholar: Author Only Title Only Author and Title

Izumi, Ishida, Nakamura, Hidema (2017) Entire Photodamaged Chloroplasts Are Transported to the Central Vacuole by Autophagy. Plant Cell 29:377-394

Google Scholar: Author Only Title Only Author and Title

Izumi, Wada, Makino, Ishida (2010) The autophagic degradation of chloroplasts via rubisco-containing bodies is specifically linked to leaf carbon status but not nitrogen status in arabidopsis. Plant Physiol 154:1196-1209

Google Scholar: Author Only Title Only Author and Title

Janse van Rensburg, Van den Ende, Signorelli (2019) Autophagy in plants: Both a puppet and a puppet master of sugars. Front Plant Sci 10:

Google Scholar: Author Only Title Only Author and Title

Jiang, Zhu, Wang, Hou (2020) Autophagy-related 2 regulates chlorophyll degradation under abiotic stress conditions in arabidopsis. Int J Mol Sci 21:4515

Google Scholar: Author Only Title Only Author and Title

Kodama (2016) Time gating of chloroplast autofluorescence allows clearer fluorescence imaging in planta. PLoS One 11:e0152484 Google Scholar: Author Only Title Only Author and Title

LaBrant, Barnes, Roston (2018) Lipid transport required to make lipids of photosynthetic membranes. Photosynth Res 138:345-360 Google Scholar: Author Only Title Only Author and Title

Lai, Wang, Zheng, Fan, Chen (2011) Acritical role of autophagy in plant resistance to necrotrophic fungal pathogens. Plant J 66:953-968 Google Scholar: Author Only Title Only Author and Title

Lastdrager, Hanson, Smeekens (2014) Sugar signals and the control of plant growth and development. J Exp Bot 65:799-807

Google Scholar: Author Only Title Only Author and Title 
Law, Narsai, Whelan (2014) Mitochondrial biogenesis in plants during seed germination. Mitochondrion 19:214-221

Google Scholar: Author Only Title Only Author and Title

Li-Beisson, Shorrosh, Beisson, Andersson, Arondel, Bates, Baud, Bird, DeBono, Durrett, et al (2013) Acyl-Lipid Metabolism In: The Arabidopsis Book. p e0161

Google Scholar: Author Only Title Only Author and Title

Li, Chung, Vierstra (2014) AUTOPHAGY-RELATED11 plays a critical role in general autophagy- and senescence-induced mitophagy in Arabidopsis. Plant Cell 26:788-807

Google Scholar: Author Only Title Only Author and Title

Li, Gao, Benning, Sharkey (2012) Characterization of photosynthesis in Arabidopsis ER-to-plastid lipid trafficking mutants. Photosynth Res 112:49-61

Google Scholar: Author Only Title Only Author and Title

Li, Yu (2018) Chloroplast galactolipids: The link between photosynthesis, chloroplast shape, jasmonates, phosphate starvation and freezing tolerance. Plant Cell Physiol 59:1128-1134

Google Scholar: Author Only Title Only Author and Title

Luo, Zhou, Masclaux-Daubresse, Wang, Wang, Zheng (2019) Morphological and physiological responses to contrasting nitrogen regimes in Populus cathayana is linked to resources allocation and carbon/nitrogen partition. Environ Exp Bot 162:247-255

Google Scholar: Author Only Title Only Author and Title

Ma, Liang, Zhao, Wang, Ma, Mai, Andrade, Zeng, Grujic, Jiang, et al (2021) Article Friendly mediates membrane depolarization- induced mitophagy in Arabidopsis. Curr Biol doi.org/10.1016/j.cub.2021.02.034.

Google Scholar: Author Only Title Only Author and Title

Marshall, Vierstra (2018) Autophagy: The Master of Bulk and Selective Recycling. Annu Rev Plant Biol 69:173-208

Google Scholar: Author Only Title Only Author and Title

Masclaux-Daubresse, Chen, Havé (2017) Regulation of nutrient recycling via autophagy. Curr Opin Plant Biol 39:8-17

Google Scholar: Author Only Title Only Author and Title

McLoughlin, Augustine, Marshall, Li, Kirkpatrick, Otegui, Vierstra (2018) Maize multi-omics reveal roles for autophagic recycling in proteome remodelling and lipid turnover. Nat Plants 4:1056-1070

Google Scholar: Author Only Title Only Author and Title

McLoughlin, Marshall, Ding, Chatt, Kirkpatrick, Augustine, Li, Otegui, Vierstra (2020) Autophagy plays prominent roles in amino acid, nucleotide, and carbohydrate metabolism during fixed-carbon starvation in maize. Plant Cell 32:2699-2724

Google Scholar: Author Only Title Only Author and Title

Michaud, Jouhet (2019) Lipid trafficking at membrane contact sites during plant development and stress response. Front Plant Sci 10:1-10

Google Scholar: Author Only Title Only Author and Title

Minina, Moschou, Vetukuri, Sanchez-Vera, Cardoso, Liu, Elander, Dalman, Beganovic, Lindberg Yilmaz, et al (2018) Transcriptional stimulation of rate-limiting components of the autophagic pathway improves plant fitness. J Exp Bot 69:1415-1432

Google Scholar: Author Only Title Only Author and Title

Nukarinen, Ngele, Pedrotti, Wurzinger, Mair, Landgraf, Börnke, Hanson, Teige, Baena-Gonzalez, et al (2016) Quantitative phosphoproteomics reveals the role of the AMPK plant ortholog SnRK1 as a metabolic master regulator under energy deprivation. Sci Rep 6:1-19

Google Scholar: Author Only Title Only Author and Title

O'Leary, Oh, Lee, Millar (2020). Metabolite regulatory interactions control plant respiratory metabolism via Target of Rapamycin (TOR) Kinase activation. Plant Cell. doi.org/10.1105/tpc.19.00157

Google Scholar: Author Only Title Only Author and Title

Pfeiffer, Janocha, Dong, Medzihradszky, Schöne, Daum, Suzaki, Forner, Langenecker, Rempel, et al (2016) Integration of light and metabolic signals for stem cell activation at the shoot apical meristem. Elife 5:

Google Scholar: Author Only Title Only Author and Title

Pogson, Ganguly, Abrecht-Borth (2015) Insights into chloroplast biogenesis and development. Biochim Biophys Acta - Bioenerg 1847:1017-1024

Google Scholar: Author Only Title Only Author and Title

Ritchie, Phipson, Wu, Hu, Law, Shi, Smyth (2015) Limma powers differential expression analyses for RNAsequencing and microarray studies. Nucleic Acids Res 43:e47

Google Scholar: Author Only Title Only Author and Title

Robinson, McCarthy, Smyth (2010) edgeR: ABioconductor package for differential expression analysis of digital gene expression data. Bioinformatics 26:139-140

Google Scholar: Author Only Title Only Author and Title 
Robinson, Oshlack (2010) Ascaling normalization method for differential expression analysis of RNAseq data. Genome Biol 11:R25 Google Scholar: Author Only Title Only Author and Title

Rudowska, Gieczewska, Mazur, Garstka, Mostowska (2012) Chloroplast biogenesis - Correlation between structure and function. Biochim Biophys Acta - Bioenerg 1817:1380-1387

Google Scholar: Author Only Title Only Author and Title

Schnurr, Shockey, De Boer, Browse (2002) Fatty acid export from the chloroplast. Molecular characterization of a major plastidial acylcoenzyme Asynthetase from Arabidopsis. Plant Physiol 129:1700-1709

Google Scholar: Author Only Title Only Author and Title

Signorelli, Tarkowski, Van den Ende, Bassham (2019) Linking Autophagy to Abiotic and Biotic Stress Responses. Trends Plant Sci 24: Google Scholar: Author Only Title Only Author and Title

Soneson, Love, Robinson (2016) Differential analyses for RNA-seq: Transcript-level estimates improve gene-level inferences [version 2; referees: 2 approved]. F1000Research 4:1521

Google Scholar: Author Only Title Only Author and Title

Thazar-Poulot, Miquel, Fobis-Loisy, Gaude (2015) Peroxisome extensions deliver the Arabidopsis SDP1 lipase to oil bodies. Proc Natl Acad Sci U S A 112:4158-4163

Google Scholar: Author Only Title Only Author and Title

Tyanova, Temu, Cox (2016) The MaxQuant computational platform for mass spectrometry-based shotgun proteomics. Nat Protoc 11:2301-2319

Google Scholar: Author Only Title Only Author and Title

Warren (2008) Rapid measurement of chlorophylls with a microplate reader. J Plant Nutr 31:1321-1332

Google Scholar: Author Only Title Only Author and Title

Waters, Langdale (2009) The making of a chloroplast. EMBO J 28:2861-2873

Google Scholar: Author Only Title Only Author and Title

Wessel, Flügge (1984) Amethod for the quantitative recovery of protein in dilute solution in the presence of detergents and lipids.

Anal Biochem 138:141-143

Google Scholar: Author Only Title Only Author and Title

Xu, Fan, Froehlich, Awai, Benning (2005) Mutation of the TGD1 chloroplast envelope protein affects phosphatidate metabolism in Arabidopsis. Plant Cell 17:3094-3110

Google Scholar: Author Only Title Only Author and Title

Xu, Fan, Riekhof, Froehlich, Benning (2003) A permease-like protein involved in ER to thylakoid lipid transfer in Arabidopsis. EMBO J 22:2370-2379

Google Scholar: Author Only Title Only Author and Title

Xu, Moellering, Muthan, Fan, Benning (2010) Lipid transport mediated by arabidopsis TGD proteins is unidirectional from the endoplasmic reticulum to the plastid. Plant Cell Physiol 51:1019-1028

Google Scholar: Author Only Title Only Author and Title

Yoshimoto, Jikumaru, Kamiya, Kusano, Consonni, Panstruga, Ohsumi, Shirasu (2009) Autophagy negatively regulates cell death by controlling NPR1-dependent salicylic acid signaling during senescence and the innate immune response in Arabidopsis. Plant Cell 21:2914-27

Google Scholar: Author Only Title Only Author and Title

Young, Bartel (2016) Pexophagy and peroxisomal protein turnover in plants. Biochim Biophys Acta - Mol Cell Res 1863:999-1005 Google Scholar: Author Only Title Only Author and Title

Zhuang, Chung, Cui, Lin, Gao, Kang, Jiang (2017) ATG9 regulates autophagosome progression from the endoplasmic reticulum in Arabidopsis. Proc Natl Acad Sci 114:E426-E435

Google Scholar: Author Only Title Only Author and Title 